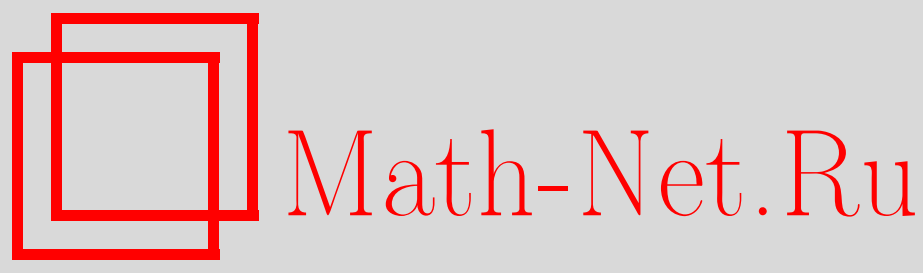

А. С. Николаев, Разложение Като в теории возмущений классической механики и явное выражение для генератора Депри, ТМФ, 2015, том 182, номер 3, 465-499

DOI: https://doi.org/10.4213/tmf8767

Использование Общероссийского математического портала Math-Net.Ru подразумевает, что вы прочитали и согласны с пользовательским соглашением http://www . mathnet.ru/rus/agreement

Параметры загрузки:

IP: 3.80 .181 .102

26 апреля 2023 г., $14: 44: 32$

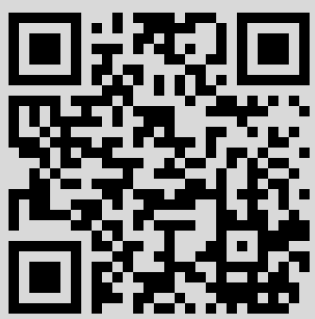




\title{
РАЗЛОЖЕНИЕ КАТО В ТЕОРИИ ВОЗМУЩЕНИЙ КЛАССИЧЕСКОЙ МЕХАНИКИ И ЯВНОЕ ВЫРАЖЕНИЕ ДЛЯ ГЕНЕРАТОРА ДЕПРИ
}

\begin{abstract}
Изучается структура рядов канонической теории возмущений ПуанкареЛиндштедта классической механики в операторной формулировке Депри и устанавливается их связь с резольвентным разложением Като. Обсуждение инвариантных определений усредняющего и интегрирующего операторов и канонических тождеств для них выявляет регулярную картину в ряде для генератора Депри. Данная регулярность объясняется при помощи рядов Като и связи пертурбативных операторов с коэффициентами Лорана резольвенты оператора Лиувилля. Этот чисто канонический подход систематизирует ряды и приводит к явному выражению для генератора Депри в любом порядке теории возмущений: $G=-\hat{\mathbf{S}}_{H} H_{j}$, где $\hat{\mathbf{S}}_{H}$ является частичным псевдообратным к возмущенному оператору Лиувилля. Соответствующий ряд Като приводит к достаточно эффективному вычислительному алгоритму. Каноническая связь возмущенного и невозмущенного усредняющих операторов позволяет описать неоднозначности генератора и преобразованного гамильтониана. При этом интегралы Густавсона оказываются нечувствительными к стилю нормализации. Непертуративные примеры используются для иллюстрации.
\end{abstract}

Ключевые слова: теория возмущений классической механики, преобразования ЛиДепри, оператор Лиувилля, резольвента, разложение Като.

DOI: $10.4213 / \operatorname{tmf} 8767$

\section{1. ВВЕДЕНИЕ}

Эта работа была инспирирована замечательными аналогиями между математическими формализмами разложений теорий возмущений классической и квантовой механик. Например, классическая каноническая секулярная теория возмущений [1] соответствует зависящей от времени квантово-механической теории возмущений [2]. Представлению “действие-угол" соответствует энергетическое представление квантовой механики. Классический метод Пуанкаре-Линдштедта [3] в ли-алгебраической формулировке [4], [5] имеет прямой аналог в квантовой теории возмущений Ван

* Институт физико-технической информатики, Протвино, Московская обл., Россия

†зАО РДТЕХ, Москва, Россия. Е-mail: Andrey.Nikolaev@rdtex.ru 
Флека [6]-[8]. Аналогично нормальные формы Биркгофа-Густавсона [9], [10] соответствуют квантово-механической теории возмущений в представлении БаргманаФока [11], [12].

Квантовая механика обладает большим разнообразием методов теории возмущений, чем классическая механика. Некоторые из этих методов могут быть интересны для классической теории возмущений. Здесь мы конструируем классический аналог ряда Като [13].

Теория возмущений Като использует разложение оператора резольвенты в ряды Неймана и Лорана в окрестности собственных значений квантово-механического гамильтониана. В классической механике нашими инструментами являются оператор Лиувилля и его резольвента. Но спектр классического оператора Лиувилля устроен сложнее, чем спектры квантовых гамильтонианов [14]. Кроме того, в многомерном случае ряд классической теории возмущений должен расходиться на всюду плотном множестве резонансов [15], [16].

Несмотря на эту внутреннюю расходимость, каноническая теория возмущений оказывается эффективным инструментом в небесной механике [17], нелинейной физике и теории ускорителей. Поэтому важны новые пертурбативные алгоритмы. В настоящей работе мы рассматриваем только формальные построения канонических пертурбативных рядов и общих выражений. Вопросы их сходимости и нерезонансные сокращения будут обсуждаться в последующих работах.

Здесь необходимо отметить, что неканонические пертурбативные разложения резольвенты оператора Лиувилля использовались в "Брюссельском формализме" неравновесной статистической механики [18]. Но мы будем конструировать чисто канонические ряды.

Изложение построено следующим образом. Вначале мы описываем конструкцию классических рядов Пуанкаре-Линдштедта-Депри, используя инвариантный операторный формализм, и вводим удобные обозначения, в основном заимствованные из квантовой механики. Затем обсуждаются алгебраические свойства базовых операторов теории возмущений. Набор канонических тождеств позволяет выявить регулярную структуру в ряде для генератора Депри.

Чтобы распространить эту структуру на все порядки, мы используем абелево среднее, взятое из квантовой механики, и устанавливаем связь с резольвентой оператора Лиувилля и разложением Като. Канонические свойства этой резольвенты позволяют получить явное выражение для генератора Депри в простом виде:

$$
G=-\hat{\mathrm{S}}_{H} H_{j}
$$

Здесь интегрирующий оператор $\hat{\mathbf{S}}_{H}$ является частичным псевдообратным к возмущенному оператору Лиувилля.

После обсуждения структуры генератора в произвольном порядке, его неоднозначности и отличия в стиле нормализации от стандартного алгоритма Депри мы распространим формулы на многомерный случай и обсудим интегралы Густавсона и вычислительную эффективность алгоритмов. Непертурбативные примеры используются для иллюстрации. 
Представленный метод комбинирует классические и квантово-механические подходы в теории возмущений. Новыми результатами являются выявленная регулярная структура в ряде для генератора, ее объяснение при помощи рядов Като, явная формула для генератора Депри в произвольном порядке и нечувствительность интегралов Густавсона к процедуре нормализации.

Набор дополнительных демонстраций может быть загружен по адресу [19]. Его файлы содержат компьютерные программы и большие выражения, включая общий вид преобразованного гамильтониана до седьмого порядка теории возмущений. Демонстрации используют свободно распространяемую систему компьютерной алгебpы FORM [20].

\section{2. КЛАССИЧЕСКАЯ ТЕОРИЯ ВОЗМУЩЕНИЙ}

2.1. Оператор Лиувилля. Уравнения движения большинства гамильтоновых систем не могут быть решены аналитически. Поэтому особый интерес представляют приближенные методы, сохраняющие фундаментальные физические структуры. Одним из таких приближений является классическая каноническая теория возмущений [21]. Она исследует динамическое поведение $\mathbf{d - м е р н о и ̆ ~ с и с т е м ы ~ с ~ г а м и л ь - ~}$ тонианом, который отличается от гамильтониана интегрируемой системы малым возмущением:

$$
H=H_{0}+\alpha H_{j}
$$

Здесь $H_{0}$ и $H_{j}$ являются функциями канонических переменных $\vec{p}, \vec{q}$ на $\mathbb{R}^{d} \times \mathbb{R}^{d}$. В данной работе рассматриваются только автономные, не зависящие от времени гамильтонианы, имеющие компактные изоэнергетические поверхности $H(\vec{p}, \vec{q})=E$. Кроме того, все функции предполагаются аналитическими.

Теория возмущений аппроксимирует временну́ю эволюцию канонических переменных и конструирует приближенные интегралы движения возмущенной системы. Ее более общей задачей является построение приближений для временно́й эволюции функции $F(\vec{p}, \vec{q})$, подчиняющейся уравнению Гамильтона:

$$
\frac{d F}{d t}=[F, H], \quad[F, H]=\sum_{j=1}^{\mathbf{d}} \frac{\partial F}{\partial q_{j}} \frac{\partial H}{\partial p_{j}}-\frac{\partial F}{\partial p_{j}} \frac{\partial H}{\partial q_{j}} .
$$

Такая эволюция определяет непрерывное однопараметрическое семейство канонических преобразований фазового пространства, называемое гамильтоновым потоком. С использованием оператора Лиувилля

$$
\hat{\mathbf{L}}_{H}=[\cdot, H], \quad \hat{\mathbf{L}}_{H} F=[F, H]
$$

формальное решение уравнения Гамильтона для не зависящего от времени гамильтониана может быть записано в виде операторной экспоненты:

$$
\left.\widetilde{F}(p, q)\right|_{t}=\left.e^{t \hat{\mathbf{L}}_{H}} F(p, q)\right|_{0}, \quad \hat{\mathbf{L}}_{H} e^{t \hat{\mathbf{L}}_{H}}=e^{t \hat{\mathbf{L}}_{H} \hat{\mathbf{L}}_{H}}
$$

Это каноническое преобразование, коммутирующее с автономным оператором $\hat{\mathbf{L}}_{H}$. 
2.2. Канонические преобразования, близкие к тождественному. Теория возмущений использует более общую конструкцию однопараметрического семейства близких к тождественному канонических преобразований фазового пространства $\mathbb{R}^{2 \mathbf{d}}$

$$
\widetilde{\mathbf{x}}=\widehat{\mathbf{U}}(\alpha) \mathbf{x}, \quad\left\{\begin{array}{l}
x_{j}=q_{j}, \\
x_{j+\mathbf{d}}=p_{j},
\end{array} \quad j=1, \ldots, \mathbf{d},\right.
$$

оснащенного канонической структурой

$$
[F(\mathbf{x}), G(\mathbf{x})]=\sum_{j, k}^{2 \mathbf{d}} \frac{\partial F}{\partial x_{j}} \omega_{j k} \frac{\partial G}{\partial x_{k}}
$$

где $\omega_{j k}=-\omega_{k j}-$ невырожденная антисимметричная матрица Якоби. Такие канонические семейства всегда являются гамильтоновыми потоками [4], [22]

$$
\frac{\partial \widehat{\mathbf{U}}}{\partial \alpha}=\hat{\mathbf{L}}_{G} \widehat{\mathbf{U}}
$$

относительно "времени" $\alpha$ с некоторым генератором $G(\mathbf{x}, \alpha)$. Теория возмущений конструирует этот генератор в виде степенного ряда $G(\mathbf{x}, \alpha)=\sum_{n=0}^{\infty} \alpha^{n} G_{n}(\mathbf{x})$. Кроме того, конструируются ряды для преобразования $\widehat{\mathbf{U}}=\sum_{n=0}^{\infty} \alpha^{n} \widehat{\mathbf{U}}_{n}$ и обратного преобразования $\widehat{\mathbf{V}}=\widehat{\mathbf{U}}^{-1}$, удовлетворяющего уравнению

$$
\frac{\partial \widehat{\mathbf{V}}}{\partial \alpha}=-\widehat{\mathbf{V}} \hat{\mathbf{L}}_{G}
$$

В небесной механике эти выражения известны как преобразования Ли-Депри [4]. Подставляя ряды для $G, \widehat{\mathbf{U}}$ и $\widehat{\mathbf{V}}$ в предыдущие формулы, Депри получил рекуррентные выражения для коэффициентов:

$$
\widehat{\mathbf{U}}_{n}=\frac{1}{n} \sum_{k=0}^{n-1} \hat{\mathbf{L}}_{G_{n-k-1}} \widehat{\mathbf{U}}_{k}, \quad \widehat{\mathbf{V}}_{n}=-\frac{1}{n} \sum_{k=0}^{n-1} \widehat{\mathbf{V}}_{k} \hat{\mathbf{L}}_{G_{n-k-1}}
$$

Итерации позволяют получить нерекуррентные выражения [1], [23]:

$$
\begin{aligned}
\widehat{\mathbf{U}}_{n} & =\sum_{\substack{\left(m_{1}, \ldots, m_{r}\right), n>m_{1}>m_{2}>\cdots>m_{r}}} \frac{\hat{\mathbf{L}}_{G_{n-m_{1}-1}}}{n} \frac{\hat{\mathbf{L}}_{G_{m_{1}-m_{2}-1}}}{m_{1}} \cdots \frac{\hat{\mathbf{L}}_{G_{m_{r}-1}}}{m_{r}}, \\
\widehat{\mathbf{V}}_{n} & =\sum_{\substack{\left(m_{1}, \ldots, m_{r}\right), m_{r} \\
n>m_{1}>m_{2}>\cdots>m_{r}}}(-1)^{r+1} \frac{\hat{\mathbf{L}}_{G_{m_{r}-1}}}{m_{r}} \cdots \frac{\hat{\mathbf{L}}_{G_{m_{1}-m_{2}-1}}}{m_{1}} \frac{\hat{\mathbf{L}}_{G_{n-m_{1}-1}}}{n} .
\end{aligned}
$$

Здесь суммирование происходит по всем наборам целых чисел $\left(m_{1}, \ldots, m_{r}\right)$, удовлетворяющих $n>m_{1}>\cdots>m_{r}>0$.

Далее мы будем использовать обозначения $\widehat{\mathbf{U}}=\exp _{\mathrm{D}}\left(\alpha \hat{\mathbf{L}}_{G}\right), \widehat{\mathbf{V}}=\exp _{\mathrm{D}}^{-1}\left(\alpha \hat{\mathbf{L}}_{G}\right)$ и термин экспоненты Депри для этих рядов, чтобы подчеркнуть их экспонентоподобную 
роль для зависящего от $\alpha$ генератора. В первых порядках

$$
\begin{aligned}
\exp _{\mathrm{D}}\left(\alpha \hat{\mathbf{L}}_{G}\right)=1 & +\alpha \hat{\mathbf{L}}_{G_{0}}+\frac{\alpha^{2}}{2}\left(\hat{\mathbf{L}}_{G_{0}}^{2}+\hat{\mathbf{L}}_{G_{1}}\right)+ \\
& +\frac{\alpha^{3}}{6}\left(\hat{\mathbf{L}}_{G_{0}}^{3}+\hat{\mathbf{L}}_{G_{0}} \hat{\mathbf{L}}_{G_{1}}+2 \hat{\mathbf{L}}_{G_{1}} \hat{\mathbf{L}}_{G_{0}}+2 \hat{\mathbf{L}}_{G_{2}}\right)+O\left(\alpha^{3}\right), \\
\exp _{\mathrm{D}}^{-1}\left(\alpha \hat{\mathbf{L}}_{G}\right)=1 & -\alpha \hat{\mathbf{L}}_{G_{0}}+\frac{\alpha^{2}}{2}\left(\hat{\mathbf{L}}_{G_{0}}^{2}-\hat{\mathbf{L}}_{G_{1}}\right)- \\
& -\frac{\alpha^{3}}{6}\left(\hat{\mathbf{L}}_{G_{0}}^{3}-2 \hat{\mathbf{L}}_{G_{0}} \hat{\mathbf{L}}_{G_{1}}-\hat{\mathbf{L}}_{G_{1}} \hat{\mathbf{L}}_{G_{0}}+2 \hat{\mathbf{L}}_{G_{2}}\right)+O\left(\alpha^{3}\right)
\end{aligned}
$$

(см. также демонстрацию “deprit_exponents").

Отметим ключевые свойства канонического преобразования $\widehat{\mathbf{U}}$. Это точечное преобразование фазового пространства $\widehat{\mathbf{U}} F(\mathbf{x})=F(\widehat{\mathbf{U}} \mathbf{x})$, которое также сохраняет скобки Пуассона. Имеет смысл рассматривать скобку Пуассона как дополнительное антисимметричное произведение (алгебра Пуассона). Канонические преобразования сохраняют как алгебраическую, так и каноническую структуры пространства функций:

$$
\begin{aligned}
\widehat{\mathbf{U}}(F H) & =(\widehat{\mathbf{U}} F)(\widehat{\mathbf{U}} H), \\
\widehat{\mathbf{U}}([F, H]) & =[\widehat{\mathbf{U}} F, \widehat{\mathbf{U}} H]
\end{aligned}
$$

для любых $F(\mathbf{x})$ и $H(\mathbf{x})$.

Согласно правилу Лейбница и тождеству Якоби оператор Лиувилля является производной по отношению к обоим произведениям:

$$
\begin{aligned}
\hat{\mathbf{L}}_{G}(F H) & =\left(\hat{\mathbf{L}}_{G} F\right) H+F\left(\hat{\mathbf{L}}_{G} H\right), \\
\hat{\mathbf{L}}_{G}[F, H] & =\left[\hat{\mathbf{L}}_{G} F, H\right]+\left[F, \hat{\mathbf{L}}_{G} H\right] .
\end{aligned}
$$

Это диалгебраическое свойство будет использоваться при рассмотрении канонических тождеств для пертурбативных операторов.

Операторный ли-алгебраический формализм генераторов канонических преобразований появился в теории возмущений классической механики в 1966 г. [24]. Этот формализм оказался существенно проще для компьютерно-алгебраических вычислений, чем подход с использованием производящих функций. Интересно, что соответствующий квантово-механический формализм возник почти за 40 лет до этого [6].

Нашей целью является каноническое преобразование гамильтониана в более простую, предпочтительно интегрируемую форму. Оказывается, преобразованный гамильтониан становится интегралом движения невозмущенной системы. Опишем кратко это стандартное построение.

Каноническое отображение $\widetilde{\mathbf{x}}=\exp _{\mathrm{D}}\left(\alpha \hat{\mathbf{L}}_{G}\right) \mathbf{x}$ преобразует возмущенный гамильтониан к виду

$$
\begin{aligned}
\widetilde{H} & =\exp _{\mathrm{D}}^{-1}\left(\alpha \hat{\mathbf{L}}_{G}\right)\left(H_{0}+\alpha H_{j}\right)=H_{0}+\alpha\left(H_{j}-\hat{\mathbf{L}}_{G_{0}} H_{0}\right)+O\left(\alpha^{2}\right)= \\
& =H_{0}+\alpha\left(\hat{\mathbf{L}}_{H_{0}} G_{0}+H_{j}\right)+\frac{\alpha^{2}}{2}\left(\hat{\mathbf{L}}_{H_{0}} G_{1}+\hat{\mathbf{L}}_{G_{0}}^{2} H_{0}-2 \hat{\mathbf{L}}_{G_{0}} H_{j}\right)+O\left(\alpha^{3}\right) .
\end{aligned}
$$

Для того чтобы упростить члены первого порядка по $\alpha$, следует обратить оператор Лиувилля $\hat{\mathbf{L}}_{H_{0}}$. Но так как этот оператор имеет непустое ядро, можно сконструировать только его частичный псевдообратный оператор. 
2.3. Базовые пертурбативные операторы. Мы предполагаем, что невозмущенная гамильтонова система, имеющая компактные изоэнергетические поверхности $H_{0}(\mathbf{x})=E$, интегрируема в смысле Лиувилля и выполняет квазипериодическое движение по инвариантным торам [21]. Тогда временно́е среднее

$$
\langle F\rangle=\left.\lim _{T \rightarrow \infty} \frac{1}{T} \int_{0}^{T} F(\vec{p}(t), \vec{q}(t))\right|_{\substack{p(0)=p, q(0)=q}} d t=\lim _{T \rightarrow \infty} \frac{1}{T} \int_{0}^{T} e^{t \hat{\mathbf{L}}_{H_{0}}} F(\mathbf{x}) d t
$$

существует в представлении "действие-угол" для любой аналитической функции $F(\mathbf{x})$. Это среднее является функцией начальной точки $\mathbf{x}$. Будучи записано в инвариантной форме, это среднее существует и в других канонических переменных.

Операция усреднения выделяет из $F(\mathbf{x})$ секулярную, неосциллирующую составляющую, которая остается постоянной при временно́й эволюции. В функциональном анализе эта операция известна как $(C, 1)$ усреднение по Чезаро [25]. Соответствующий оператор

$$
\hat{\mathbf{P}}_{H_{0}}=\lim _{T \rightarrow \infty} \frac{1}{T} \int_{0}^{T} e^{t \hat{\mathbf{L}}_{H_{0}}} d t
$$

является проектором $\hat{\mathbf{P}}_{H_{0}}^{2}=\hat{\mathbf{P}}_{H_{0}}$. Он проецирует $F(\mathbf{x})$ на секулярное пространство функций, коммутативных с $H_{0}$. Это пространство является ядром оператора $\hat{\mathbf{L}}_{H_{0}}$ и алгеброй интегралов движения невозмущенного гамильтониана $H_{0}$.

Обозначение $\hat{\mathbf{P}}_{H_{0}}$ взято из квантовой механики. Стандартные обозначения, такие как $\langle\cdot\rangle, \overline{\mathbf{F}}$ и $M_{t}[26],[27]$ не подчеркивают нужную нам структуру в пертурбативных разложениях.

Комплементарный проектор $1-\hat{\mathbf{P}}_{H_{0}}$ выделяет из $F(\mathbf{x})$ осциллирующую часть. Он проецирует на несекулярное пространство осциллирующих функций, где существует оператор, обратный к оператору $\hat{\mathbf{L}}_{H_{0}}$ (здесь рассматриваются только полупростые $\hat{\mathbf{L}}_{H_{0}}$ ).

Этот интегрирующий оператор известен также как решение гомологического уравнения, тильда-операция, первообразная с нулевым средним [28], $\widehat{\Gamma}$-операция Фридрихса [29], 1/k-оператор и т. д. Его инвариантное определение [26]:

$$
\hat{\mathbf{S}}_{H_{0}}=-\lim _{T \rightarrow \infty} \frac{1}{T} \int_{0}^{T} d t \int_{0}^{t} d \tau e^{\tau \hat{\mathbf{L}}_{H_{0}}}\left(1-\hat{\mathbf{P}}_{H_{0}}\right) .
$$

Выражения, явно учитывающие квазипериодичность движения, можно найти в [30], [31]. Формальное вычисление

$$
\begin{aligned}
\hat{\mathbf{L}}_{H_{0}} \hat{\mathbf{S}}_{H_{0}} & =-\lim _{T \rightarrow \infty} \frac{1}{T} \int_{0}^{T} d t \int_{0}^{t} d \tau \frac{\partial}{\partial \tau} e^{\tau \hat{\mathbf{L}}_{H_{0}}}\left(1-\hat{\mathbf{P}}_{H_{0}}\right)= \\
& =-\lim _{T \rightarrow \infty} \frac{1}{T} \int_{0}^{T} d t\left(e^{t \hat{\mathbf{L}}_{H_{0}}}-1\right)\left(1-\hat{\mathbf{P}}_{H_{0}}\right)=1-\hat{\mathbf{P}}_{H_{0}}
\end{aligned}
$$


демонстрирует, что оператор $\hat{\mathbf{S}}_{H_{0}}$ является частичным псевдообратным к невозмущенному оператору Лиувилля:

$$
\begin{aligned}
\hat{\mathbf{L}}_{H_{0}} \hat{\mathbf{S}}_{H_{0}} & =\hat{\mathbf{S}}_{H_{0}} \hat{\mathbf{L}}_{H_{0}}=1-\hat{\mathbf{P}}_{H_{0}}, \\
\hat{\mathbf{S}}_{H_{0}} \hat{\mathbf{P}}_{H_{0}} & =\hat{\mathbf{P}}_{H_{0}} \hat{\mathbf{S}}_{H_{0}} \equiv 0 .
\end{aligned}
$$

Эти три базовых оператора $\hat{\mathbf{L}}_{H_{j}}, \hat{\mathbf{P}}_{H_{0}}$ и $\hat{\mathbf{S}}_{H_{0}}$ являются строительными блоками пертурбативных разложений. Теория возмущений классической механики дает несколько алгоритмов их вычисления.

1. Представление "действие-угол" [21]. В канонических координатах "действиеугол" невозмущенный гамильтониан является функцией только $\mathbf{d}$ переменных “действие" $\vec{J}$, а возмущение $2 \pi$-периодично по фазам $\vec{\phi}$ :

$$
H=H_{0}(\vec{J})+\alpha H_{j}(\vec{J}, \vec{\phi})=H_{0}(\vec{J})+\alpha \sum_{\vec{k}} \widetilde{H}_{j}(\vec{J}, \vec{k}) e^{i(\vec{k}, \vec{\phi})}
$$

Движение невозмущенной системы квазипериодично:

$$
\vec{J}=\text { const }, \quad \vec{\phi}(t)=\vec{\omega} t+\vec{\phi}_{0}, \quad \vec{\omega}=\frac{\partial H_{0}}{\partial \vec{J}} .
$$

Поскольку фурье-компоненты в представлении “действие-угол" являются собственными функциями $\hat{\mathbf{L}}_{H_{0}}$, пертурбативные операторы могут быть записаны как

$$
\begin{aligned}
\hat{\mathbf{P}}_{H_{0}} F & =\lim _{T \rightarrow \infty} \frac{1}{T} \int_{0}^{T} d t \sum_{\vec{k}} \widetilde{F}(\vec{J}, \vec{k}) e^{i\left(\vec{k}, \vec{\phi}_{0}\right)+i(\vec{\omega}, \vec{k}) t}=\sum_{(\vec{\omega}, \vec{k})=0} \widetilde{F}(\vec{J}, \vec{k}) e^{i\left(\vec{k}, \vec{\phi}_{0}\right)}, \\
\hat{\mathbf{S}}_{H_{0}} F & =\sum_{(\vec{\omega}, \vec{k}) \neq 0} \frac{1}{i(\vec{\omega}, \vec{k})} \widetilde{F}(\vec{J}, \vec{k}) e^{i\left(\vec{k}, \vec{\phi}_{0}\right)} .
\end{aligned}
$$

Часто эти хорошо известные выражения используются в качестве определений усредняющего и интегрирующего операторов.

2. Нормальные формы Биркгофа-Густавсона-Брюно [9], [10], [32] для степенных рядов. В простейшем случае квадратичный невозмущенный гамильтониан может быть приведен к виду

$$
H_{0}=\sum_{k=1}^{\mathbf{d}} \frac{\omega_{k}}{2}\left(p_{k}^{2}+q_{k}^{2}\right)
$$

В результате канонического преобразования к комплексным координатам (аналогам $\hat{a}, \hat{a}^{\dagger}$ в квантовой механике)

$$
q_{k}=\frac{1}{\sqrt{2}}\left(\xi_{k}+i \eta_{k}\right), \quad p_{k}=\frac{i}{\sqrt{2}}\left(\xi_{k}-i \eta_{k}\right)
$$

возмущенный гамильтониан приобретает вид

$$
H=\sum_{k=1}^{\mathbf{d}} i \omega_{k} \xi_{k} \eta_{k}+\alpha \sum_{|\vec{m}|+|\vec{n}| \geqslant 3} \widetilde{H}_{j}(\vec{m}, \vec{n}) \prod_{k=1}^{\mathbf{d}} \xi_{k}^{m_{k}} \eta_{k}^{n_{k}} .
$$


Мономы $\xi^{\vec{m}} \eta^{\vec{n}}=\prod \xi_{k}^{m_{k}} \eta_{k}^{n_{k}}$ являются собственными векторами невозмущенного оператора Лиувилля

$$
\hat{\mathbf{L}}_{H_{0}} \xi^{\vec{m}} \eta^{\vec{n}}=i(\vec{\omega}, \vec{m}-\vec{n}) \xi^{\vec{m}} \eta^{\vec{n}} .
$$

А следовательно, для любого ряда $F(\vec{p}, \vec{q})=\sum \widetilde{F}(\vec{m}, \vec{n}) \xi^{\vec{m}} \eta^{\vec{n}}$ выполняются соотношения

$$
\begin{aligned}
\hat{\mathbf{P}}_{H_{0}} F & =\sum_{(\vec{\omega}, \vec{m}-\vec{n})=0} F(\vec{m}, \vec{n}) \xi^{\vec{m}} \eta^{\vec{n}}, \\
\hat{\mathbf{S}}_{H_{0}} F & =\sum_{(\vec{\omega}, \vec{m}-\vec{n}) \neq 0} \frac{1}{i(\vec{\omega}, \vec{m}-\vec{n})} F(\vec{m}, \vec{n}) \xi^{\vec{m}} \eta^{\vec{n}} .
\end{aligned}
$$

Отметим, что это только небольшая экскурсия в теорию нормальных форм [32], [28].

3. Алгебраические подходы [33], [34]. Гомологическое уравнение решается матричными методами в обертывающей алгебре.

4. Квадратура Журавлева [35], [36]. Функция $F(\mathbf{x})$ интегрируется вдоль невозмущенных траекторий $\mathbf{x}\left(t, \mathbf{x}_{\mathbf{0}}\right)$. Асимптотика одной квадратуры

$$
\left.\int_{0}^{T} F\left(\vec{p}\left(t, \vec{q}_{0}, \vec{p}_{0}\right)\right), \vec{q}\left(t, \vec{q}_{0}, \vec{p}_{0}\right)\right) d t, \quad T \rightarrow \infty,
$$

позволяет найти и $\hat{\mathbf{P}}_{H_{0}} F$, и $\hat{\mathbf{S}}_{H_{0}} F$ как части, соответствующие $O(T)$ и $O(1)$. Эта квадратура оказывается чрезвычайно эффективной в теории нормальных форм.

2.4. Ряды Депри. Если в выражении (4) выбрать $G_{0}=-\hat{\mathbf{S}}_{H_{0}} H_{j}$, то все члены порядка $\alpha$ в преобразованном гамильтониане станут секулярными (будут начинаться c $\left.\hat{\mathbf{P}}_{H_{0}}\right)$ :

$$
\widetilde{H}=\exp _{\mathrm{D}}^{-1}\left(\alpha \hat{\mathbf{L}}_{G}\right)\left(H_{0}+\alpha H_{j}\right)=H_{0}+\alpha \hat{\mathbf{P}} H_{j}+\frac{\alpha^{2}}{2}\left(\hat{\mathbf{L}}_{H_{0}} G_{1}+\hat{\mathbf{L}}_{\hat{\mathbf{s}} H_{j}}(1+\hat{\mathbf{P}}) H_{j}\right)+O\left(\alpha^{3}\right) .
$$

Здесь и далее мы будем опускать индекс $H_{0}$ у невозмущенных операторов $\hat{\mathbf{P}}_{H_{0}}$ и $\hat{\mathbf{S}}_{H_{0}}$.

В следующих порядках можно последовательно выбирать $G_{1}=-\hat{\mathbf{S}} \hat{\mathbf{L}}_{\hat{\mathbf{S}} H_{j}}(1+\hat{\mathbf{P}}) H_{j}$, чтобы сократить несекулярные члены порядка $O\left(\alpha^{2}\right)$, затем $G_{2}$, чтобы сократить несекулярные члены $O\left(\alpha^{3}\right)$, и т. д. Мы будем ссылаться на этот процесс как на программу классической теории возмущений Пуанкаре-Линдштедта: при помощи близкого к тождественному канонического преобразования превратить гамильтониан в интеграл невозмущенной системы.

Сравним это с программой квантово-механической теории возмущений: nри помощи близкого $\kappa$ тождественному унитарного преобразования превратить гамильтониан в блочно-диагональный оператор (коммутативный с невозмущенным гамильтонианом).

Описанная процедура рекуррентно конструирует генератор нормализующего преобразования до любого порядка по $\alpha$. Детали “треугольного” алгоритма Депри можно найти в классических книгах по теории возмущений [37], [38]. В настоящей работе мы сосредоточимся на поиске регулярностей в пертурбативных рядах. В первых 
порядках генератор и нормализованный гамильтониан имеет вид

$$
\begin{aligned}
G= & -\hat{\mathbf{S}} H_{j}-\alpha \hat{\mathbf{S}} \hat{\mathbf{L}}_{\hat{\mathbf{S}} H_{j}}(1+\hat{\mathbf{P}}) H_{j}- \\
& -\alpha^{2} \hat{\mathbf{S}}\left(\frac{1}{2} \hat{\mathbf{L}}_{\hat{\mathbf{S}} H_{j}} \hat{\mathbf{L}}_{\hat{\mathbf{P}} H_{j}}+\left(\hat{\mathbf{L}}_{H_{j}} \hat{\mathbf{S}}-\hat{\mathbf{L}}_{\hat{\mathbf{S}} H_{j}} \hat{\mathbf{P}}+\frac{1}{2} \hat{\mathbf{L}}_{\hat{\mathbf{P}} H_{j}} \hat{\mathbf{S}}\right) \hat{\mathbf{L}}_{(1+\hat{\mathbf{P}}) H_{j}}\right) \hat{\mathbf{S}} H_{j}+O\left(\alpha^{3}\right), \\
\widetilde{H}= & H_{0}+\alpha \hat{\mathbf{P}} H_{j}+\frac{\alpha^{2}}{2} \hat{\mathbf{P}} \hat{\mathbf{L}}_{\hat{\mathbf{S}} H_{j}}(1+\hat{\mathbf{P}}) H_{j}+ \\
& +\frac{\alpha^{3}}{3} \hat{\mathbf{P}}\left(\frac{1}{2} \hat{\mathbf{L}}_{\hat{\mathbf{S}} H_{j}} \hat{\mathbf{L}}_{\hat{\mathbf{P}} H_{j}}+\left(\hat{\mathbf{L}}_{H_{j}} \hat{\mathbf{S}}-\hat{\mathbf{L}}_{\hat{\mathbf{S}} H_{j}} \hat{\mathbf{P}}+\frac{1}{2} \hat{\mathbf{L}}_{\hat{\mathbf{P}} H_{j}} \hat{\mathbf{S}}\right) \hat{\mathbf{L}}_{(1+\hat{\mathbf{P}}) H_{j}}\right) \hat{\mathbf{S}} H_{j}+O\left(\alpha^{3}\right) .
\end{aligned}
$$

На первый взгляд, в этих выражениях нет заметной структуры. Но далее при помощи канонических тождеств формулы будут приведены к более систематизированному виду.

Выбор генератора нормализующего преобразования, очевидно, не уникален. Произвольная секулярная функция может быть добавлена в любом порядке. Все последующие порядки будут зависеть от этого слагаемого. Конкретное правило выбора секулярной части $G$ называется стилем (гипер)нормализации [28]. Обычно конструируют полностью несекулярный генератор преобразования Пуанкаре-Линдштедта $\hat{\mathbf{P}}_{H_{0}} G=0$. Но мы увидим, что могут быть полезны и другие условия.

Скажем несколько слов об интегрируемости нормализованной системы. В нерезонансном случае невозмущенная интегрируемая $\mathbf{d}$-мерная система имеет только $\mathbf{d}$ интегралов движения. Нормализованный возмущенный гамильтониан будет функцией только этих “действий” и формально интегрируем (до $O\left(\alpha^{n}\right)$ ).

Однако если частоты невозмущенной системы связаны $\mathbf{r}$ независимыми резонансными соотношениями $\left(\vec{\omega}, \vec{D}_{k}\right)=0, k=1, \ldots, \mathbf{r}$, то такая система имеет $\mathbf{r}$ дополнительных некоммутативных интегралов движения. Для возмущенной системы все еще можно выбрать $\mathbf{d}$ независимых инволютивных “действий”, но нормализованный гамильтониан будет зависеть и от фаз. Даже в этом так называемом резонансном случае удается понизить размерность нормализованной системы на величину размерности центра алгебры невозмущенных интегралов. Этот центр состоит из $\mathbf{d}-\mathbf{r}$ коммутативных со всеми другими интегралов [10]. Редукция по этим переменным [21], [39] приводит к r-мерной эффективной системе. Следовательно, в случае двух и более резонансов нормализованная система будет, вообще говоря, неинтегрируема.

Отметим здесь различие между классической и квантово-механической теориями возмущений. В квантовой механике возможна полная диагонализация гамильтониана с любым числом резонансных соотношений. Дополнительные квантово-механические интегралы и связь диагонализующих преобразований с нелинейными обобщениями конечномерных преобразований Боголюбова обсуждались в работах [12], [40].

\section{3. КАНОНИЧЕСКИЕ ТОЖДЕСТВА}

Рассмотрим алгебраические свойства базовых операторов $\hat{\mathbf{L}}, \hat{\mathbf{P}}_{H_{0}}$ и $\hat{\mathbf{S}}_{H_{0}}$.

1. Инвариантные определения (6), (7) операторов $\hat{\mathbf{P}}$ и $\hat{\mathbf{S}}$ приводят к равенствам

$$
\begin{aligned}
\hat{\mathbf{P}} H_{0} & =H_{0}, \quad \hat{\mathbf{S}} H_{0}=0, \\
\hat{\mathbf{P}} \hat{\mathbf{L}}_{H_{0}} & =\hat{\mathbf{L}}_{H_{0}} \hat{\mathbf{P}}=0, \\
\hat{\mathbf{S}} \hat{\mathbf{L}}_{H_{0}} & =\hat{\mathbf{L}}_{H_{0}} \hat{\mathbf{S}}=1-\hat{\mathbf{P}} .
\end{aligned}
$$


2. Так как канонические преобразования сохраняют и алгебраическое произведение, и скобки Пуассона, то для любых функций $F(\mathbf{x}), G(\mathbf{x})$ и $H_{j}(\mathbf{x})$

$$
\begin{aligned}
\hat{\mathbf{P}}(F \cdot \hat{\mathbf{P}} G) & =(\hat{\mathbf{P}} F) \cdot(\hat{\mathbf{P}} G), \\
\hat{\mathbf{P}} \hat{\mathbf{L}}_{\hat{\mathbf{P}} H_{j}} & =\hat{\mathbf{L}}_{\hat{\mathbf{P}} H_{j}} \hat{\mathbf{P}}, \\
\hat{\mathbf{S}}(F \cdot \hat{\mathbf{P}} G) & =(\hat{\mathbf{S}} F) \cdot(\hat{\mathbf{P}} G), \\
\hat{\mathbf{S}} \hat{\mathbf{L}}_{\hat{\mathbf{P}} H_{j}} & =\hat{\mathbf{L}}_{\hat{\mathbf{P}} H_{j}} \hat{\mathbf{S}} .
\end{aligned}
$$

Первые два тождества демонстрируют, что проектор $\hat{\mathbf{P}}$ сохраняет произведения, если один из операндов принадлежит ядру $\hat{\mathbf{L}}_{H_{0}}$. Это канонический аналог ортогонального проецирования.

3. Так как операция $\hat{\mathbf{L}}$ является дифференцированием и алгебраического, и пуассонова произведений функций, то существуют и формулы интегрирования по частям - тождества Фридрихса [29]

$$
\begin{aligned}
\hat{\mathbf{S}} \hat{\mathbf{L}}_{H_{j}} \hat{\mathbf{S}}= & \hat{\mathbf{L}}_{\hat{\mathbf{P}} H_{j}} \hat{\mathbf{S}}^{2}+\hat{\mathbf{L}}_{\hat{\mathbf{S}} H_{j}} \hat{\mathbf{S}}-\hat{\mathbf{S}} \hat{\mathbf{L}}_{\hat{\mathbf{S}}_{H_{j}}}-\hat{\mathbf{P}} \hat{\mathbf{L}}_{\hat{\mathbf{S}} H_{j}} \hat{\mathbf{S}}+\hat{\mathbf{S}}_{\hat{\mathbf{L}}_{\hat{\mathbf{S}} H_{j}}} \hat{\mathbf{P}}, \\
\hat{\mathbf{S}}(F \cdot \hat{\mathbf{S}} G)= & \hat{\mathbf{P}} F \cdot \hat{\mathbf{S}}^{2} G+(\hat{\mathbf{S}} F \cdot \hat{\mathbf{S}} G)-\hat{\mathbf{S}}(\hat{\mathbf{S}} F \cdot G)- \\
& -\hat{\mathbf{P}}(\hat{\mathbf{S}} F \cdot \hat{\mathbf{S}} G)+\hat{\mathbf{S}}(\hat{\mathbf{S}} F \cdot \hat{\mathbf{P}} G) .
\end{aligned}
$$

Для доказательства следует подействовать обеими частями $\hat{\mathbf{S}} \hat{\mathbf{L}}_{H_{0}}=1-\hat{\mathbf{P}}$ на равенство $\hat{\mathbf{L}}_{\hat{\mathbf{S}} H_{j}} \hat{\mathbf{S}} F=\left[\hat{\mathbf{S}} F, \hat{\mathbf{S}} H_{j}\right]$ (или $\hat{\mathbf{S}} F \cdot \hat{\mathbf{S}} G$ ) и раскрыть тождество Якоби (правило Лейбница).

Можно достичь полной симметрии формул, обозначая алгебраическое произведение как оператор. Ниже мы даже не будем приводить тождества для алгебраического произведения, автоматически предполагая существование комплементарного тождества.

4. Дальнейшие тождества существуют для произведений трех базовых операторов. Для любой функции $H_{j}$

$$
\begin{aligned}
& \hat{\mathbf{P}} \hat{\mathbf{L}}_{H_{j}} \hat{\mathbf{P}}=\hat{\mathbf{L}}_{\hat{\mathbf{P}} H_{j}} \hat{\mathbf{P}}, \\
& \hat{\mathbf{S}} \hat{\mathbf{L}}_{H_{j}} \hat{\mathbf{P}}=\hat{\mathbf{L}}_{\hat{\mathbf{s}} H_{j}} \hat{\mathbf{P}} .
\end{aligned}
$$

Это следствие инвариантности скобок Пуассона при канонических преобразованиях:

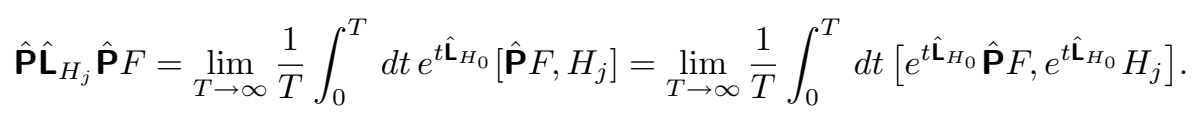

Так как $\hat{\mathbf{P}} F$ является интегралом движения $H_{0}$, имеем

$$
\hat{\mathbf{P}} \hat{\mathbf{L}}_{H_{j}} \hat{\mathbf{P}} F=\left[\hat{\mathbf{P}} F, \lim _{T \rightarrow \infty} \frac{1}{T} \int_{0}^{T} d t e^{t \hat{\mathbf{L}}_{H_{0}} H_{j}}\right]=\hat{\mathbf{L}}_{\hat{\mathbf{P}} H_{j}} \hat{\mathbf{P}} F
$$

Доказательство второго тождества аналогично.

5. Тождество Бурштейна-Соловьева [41]

$$
\hat{\mathbf{P}} \hat{\mathbf{L}}_{H_{j}} \hat{\mathbf{S}}=-\hat{\mathbf{P}} \hat{\mathbf{L}}_{\hat{\mathbf{S}} H_{j}}
$$


следует из того, что выражение $\hat{\mathbf{P}} \hat{\mathbf{L}}_{H_{0}} \hat{\mathbf{L}}_{\hat{\mathbf{S}}_{H_{j}}} \hat{\mathbf{S}}$ тождественно равно нулю. Используя тождество Якоби $\hat{\mathbf{L}}_{F} \hat{\mathbf{L}}_{G}-\hat{\mathbf{L}}_{G} \hat{\mathbf{L}}_{F}=\hat{\mathbf{L}}_{\hat{\mathbf{L}}_{F} G}$, получим

$$
0 \equiv \hat{\mathbf{P}} \hat{\mathbf{L}}_{H_{0}} \hat{\mathbf{L}}_{\hat{\mathbf{S}} H_{j}} \hat{\mathbf{S}}=\hat{\mathbf{P}} \hat{\mathbf{L}}_{\hat{\mathbf{S}} H_{j}} \hat{\mathbf{L}}_{H_{0}} \hat{\mathbf{S}}+\hat{\mathbf{P}} \hat{\mathbf{L}}_{\hat{\mathbf{L}}_{H_{0}} \hat{\mathbf{S}} H_{j}} \hat{\mathbf{S}}=\hat{\mathbf{P}} \hat{\mathbf{L}}_{\hat{\mathbf{S}}_{H_{j}}}+\hat{\mathbf{P}} \hat{\mathbf{L}}_{H_{j}} \hat{\mathbf{S}}-\hat{\mathbf{P}} \hat{\mathbf{L}}_{\hat{\mathbf{S}} H_{j}} \hat{\mathbf{P}}-\hat{\mathbf{P}} \hat{\mathbf{L}}_{\hat{\mathbf{P}} H_{j}} \hat{\mathbf{S}}
$$

Два последних члена здесь исчезают согласно (14) и (12). Таким образом, $\hat{\mathbf{P}}_{\hat{\mathbf{s}} H_{j}}+$ $\hat{\mathbf{P}} \hat{\mathbf{L}}_{H_{j}} \hat{\mathbf{S}} \equiv 0$.

Оказывается, тождеств (11)-(15) нам будет достаточно для упрощения рядов Депри. Их легко также проверить в представлении “действие-угол". Ниже мы получим и их обобщение.

Для автоматизации работы с перечисленными выражениями мы использовали систему компьютерной алгебры FORM [20]. Каноническими тождествами первые порядки рядов Депри (10) приводятся к очень интересному виду (см. также демонстрацию "deprit_classic"):

$$
\begin{aligned}
G= & -\hat{\mathbf{S}} H_{j}+\alpha\left(\hat{\mathbf{S}} \hat{\mathbf{L}} \hat{\mathbf{S}} H_{j}-\hat{\mathbf{S}}^{2} \hat{\mathbf{L}} \hat{\mathbf{P}} H_{j}\right)+\alpha^{2}\left(-\hat{\mathbf{S}} \hat{\mathbf{L}} \hat{\mathbf{S}} \hat{\mathbf{S}} H_{j}+\right. \\
& \left.+\hat{\mathbf{S}}^{\hat{\mathbf{L}}^{2}} \hat{\mathbf{L}} \hat{\mathbf{P}} H_{j}+\hat{\mathbf{S}}^{2} \hat{\mathbf{L}} \hat{\mathbf{S}} \hat{\mathbf{L}} H_{j}+\hat{\mathbf{S}}^{2} \hat{\mathbf{L}} \hat{\mathbf{P}} \hat{\mathbf{S}} H_{j}\right)+O\left(\alpha^{3}\right), \\
\widetilde{H}= & H_{0}+\alpha \hat{\mathbf{P}} H_{j}-\frac{\alpha^{2}}{2} \hat{\mathbf{P}} \hat{\mathbf{L}} \hat{\mathbf{S}} H_{j}+\alpha^{3}\left(\frac{1}{3} \hat{\mathbf{P}} \hat{\mathbf{L}} \hat{\mathbf{S}} \hat{\mathbf{S}} H_{j}-\frac{1}{6} \hat{\mathbf{P}} \hat{\mathbf{L}} \hat{\mathbf{S}}^{2} \hat{\mathbf{L}} \hat{\mathbf{P}} H_{j}\right)+O\left(\alpha^{4}\right) .
\end{aligned}
$$

Выражение для преобразованного гамильтониана соответствует классическому результату Бурштейна-Соловьева [41], [26]. В стандартных обозначениях преобразованный гамильтониан имеет следующий вид:

$$
\widetilde{H}=H_{0}+\alpha \bar{H}_{j}+\frac{\alpha^{2}}{2} \overline{\left[\widetilde{H}_{j}, H_{j}\right]}+\frac{\alpha^{3}}{3} \overline{\left[\widetilde{H}_{j},\left[\widetilde{H}_{j}, H_{j}+\frac{\bar{H}_{j}}{2}\right]\right]}+O\left(\alpha^{4}\right) .
$$

А выражение для генератора похоже на сумму всех возможных расстановок операторов $-\hat{\mathbf{S}}$ и $\hat{\mathbf{P}}$. В третьем порядке выражение для генератора Депри при выборе несекулярного стиля нормализации теряет этот простой вид. Но было замечено, что такая же сумма по всем расстановкам нормализует гамильтониан и в следующих порядках. Это стоит исследовать более детально.

\section{4. РАЗЛОЖЕНИЯ КАТО}

4.1. Резольвента оператора Лиувилля. Квантовая механика обычно использует более сильное абелево среднее [25], которое применимо и к классическому случаю:

$$
\langle F\rangle^{(A)}=\lim _{\lambda \rightarrow+0} \lambda \int_{0}^{+\infty} e^{-\lambda t} e^{t \hat{\mathbf{L}}_{H_{0}}} F(\mathbf{x}) d t .
$$

Соответствующие усредняющий и интегрирующий операторы известны из квантовой механики. Их можно записать в виде [42]-[44]

$$
\begin{aligned}
& \hat{\mathbf{P}}_{H_{0}}=\lim _{\lambda \rightarrow+0} \lambda \int_{0}^{+\infty} d t e^{-\lambda t} e^{t \hat{\mathbf{L}}_{H_{0}}}, \\
& \hat{\mathbf{S}}_{H_{0}}=-\lim _{\lambda \rightarrow+0} \int_{0}^{\infty} d t e^{-\lambda t} e^{t \hat{\mathbf{L}}_{H_{0}}}\left(1-\hat{\mathbf{P}}_{H_{0}}\right) .
\end{aligned}
$$


Если среднее по Чезаро существует, оно совпадает со средним по Абелю [25]. Поэтому мы будем использовать те же обозначения. Ниже мы будем подразумевать абелево среднее, если не оговорено другое. Это существенно упрощает формулы и позволяет естественный переход к резольвентному формализму. Строго говоря, следовало бы обсудить соответствующие тауберовы теоремы, но мы ограничимся получением формальных разложений.

Абелевы определения для $\hat{\mathbf{S}}$ и $\hat{\mathbf{P}}$, а также квантово-механические аналогии подсказывают исследование резольвенты оператора Лиувилля

$$
\hat{\mathcal{R}}_{H}(z)=\frac{1}{\hat{\mathbf{L}}_{H}-z} \text {. }
$$

Эта операторнозначная функция комплексного переменного $z$ является преобразованием Лапласа оператора эволюции гамильтоновой системы

$$
\hat{\boldsymbol{\mathcal { R }}}_{H}(z)=-\int_{0}^{+\infty} d t e^{-z t} e^{t \hat{\mathbf{L}}_{H}}
$$

Сингулярности резольвенты являются собственными значениями оператора Лиувилля $\hat{\mathbf{L}}_{H}$. Для системы с компактными изоэнергетическими поверхностями эти собственные значения лежат на мнимой оси. Обычно спектр оператора Лиувилля всюду плотен [14].

Мы начнем с более простого примера чисто дискретного спектра. Рассмотрим одномерный случай и ограничим область определения резольвенты аналитическими функциями с аргументом, принадлежащим компактной изоэнергетической поверхности $H(\mathbf{x})=E$. В таких условиях система не является перемешивающей и осциллирует с единственной частотой $\omega(E)$. Сингулярности такой резольвенты расположены в изолированных точках $0, \pm i \omega, \pm 2 i \omega, \ldots$.

Рассмотрим аналитическую структуру резольвенты в окрестности начала координат. Существование операторов $\hat{\mathbf{P}}_{H_{0}}$ и $\hat{\mathbf{S}}_{H_{0}}$ (17) означает, что невозмущенная резольвента имеет простой полюс в нуле. Усредняющий оператор есть вычет резольвенты в этом полюсе:

$$
\hat{\mathbf{P}}_{H_{0}} \equiv-\operatorname{Res}_{z=0} \hat{\mathcal{R}}_{H_{0}}
$$

а интегрирующий оператор является ее голоморфной частью:

$$
\hat{\mathbf{S}}_{H_{0}}=\lim _{z \rightarrow 0} \hat{\boldsymbol{R}}_{H_{0}}(z)\left(1-\hat{\mathbf{P}}_{H_{0}}\right) .
$$

Таким образом, резольвента объединяет оба базовых пертурбативных оператоpa [45].

Нам также потребуется резольвентное тождество Гильберта

$$
\hat{\mathcal{R}}_{H}\left(z_{1}\right)-\hat{\boldsymbol{\mathcal { R }}}_{H}\left(z_{2}\right)=\left(z_{1}-z_{2}\right) \hat{\boldsymbol{\mathcal { R }}}_{H}\left(z_{1}\right) \hat{\boldsymbol{\mathcal { R }}}_{H}\left(z_{2}\right),
$$

которое выполняется для любых комплексных $z_{1}$ и $z_{2}$ вне спектра (резольвентного множества) $\hat{\mathbf{L}}_{H}$. 
4.1.1. Ряд Лорана. Хотя невозмущенная резольвента оператора Лиувилля имеет простой полюс в начале координат, возмущенная резольвента может быть более сингулярна. Для необходимой общности запишем ряд Лорана для резольвенты с изолированной особенностью в начале координат в виде

$$
\hat{\mathcal{R}}_{H}(z)=\sum_{n=-\infty}^{+\infty} \hat{\mathbf{R}}_{H}^{(n)} z^{n-1}, \quad \hat{\mathbf{R}}_{H}^{(n)}=\frac{1}{2 \pi i} \oint_{|z|=\epsilon} \hat{\mathcal{R}}_{H}(z) z^{-n} d z
$$

(здесь обозначение $(n)$ - индекс).

Хорошо известно, что тождество Гильберта (19) определяет структуру этого ряда [13]. Имеет смысл повторить здесь этот вывод. Рассмотрим произведение двух коэффициентов

$$
\begin{aligned}
\hat{\mathbf{R}}_{H}^{(m)} \hat{\mathbf{R}}_{H}^{(n)} & =\left(\frac{1}{2 \pi i}\right)^{2} \oint_{\left|z_{1}\right|=\epsilon_{1}} \oint_{\left|z_{2}\right|=\epsilon_{2}} \hat{\mathcal{R}}_{H}\left(z_{1}\right) \hat{\mathcal{R}}_{H}\left(z_{2}\right) z_{1}^{-m} z_{2}^{-n} d z_{1} d z_{2}= \\
& =\left(\frac{1}{2 \pi i}\right)^{2} \oint_{\left|z_{1}\right|=\epsilon_{1}} \oint_{\left|z_{2}\right|=\epsilon_{2}} \frac{z_{1}^{-m} z_{2}^{-n}}{z_{2}-z_{1}}\left(\hat{\mathcal{R}}_{H}\left(z_{2}\right)-\hat{\mathcal{R}}_{H}\left(z_{1}\right)\right) d z_{1} d z_{2}
\end{aligned}
$$

Интегрирование по двум окружностям радиусов $\epsilon_{1}<\epsilon_{2}<\omega$ дает

$$
\begin{aligned}
& \frac{1}{2 \pi i} \oint_{\left|z_{1}\right|=\epsilon_{1}} \frac{z_{1}^{-m}}{z_{2}-z_{1}} d z_{1}=\eta_{m} z_{2}^{-m} \\
& \frac{1}{2 \pi i} \oint_{\left|z_{2}\right|=\epsilon_{2}} \frac{z_{2}^{-n}}{z_{2}-z_{1}} d z_{2}=\left(1-\eta_{n}\right) z_{1}^{-n}, \quad \eta_{n}=\left\{\begin{array}{l}
1 \\
0 \quad \text { при } n<1 .
\end{array}\right.
\end{aligned}
$$

В результате операторные коэффициенты резольвенты должны подчиняться соотношению

$$
\hat{\mathbf{R}}_{H}^{(m)} \hat{\mathbf{R}}_{H}^{(n)}=\left(\eta_{m}+\eta_{n}-1\right) \hat{\mathbf{R}}_{H}^{(m+n)} .
$$

Для $n=m=0$ это означает, что вычет резольвенты (со знаком минус) есть проектор

$$
\hat{\mathbf{R}}_{H}^{(0)}=-\hat{\mathbf{R}}_{H}^{(0)} \hat{\mathbf{R}}_{H}^{(0)}=-\hat{\mathbf{P}}_{H}
$$

и

$$
\begin{aligned}
& \hat{\mathbf{R}}_{H}^{(n)}=\hat{\mathbf{S}}_{H}^{n}, \quad n \geqslant 1, \\
& \hat{\mathbf{R}}_{H}^{(-n)}=-\hat{\mathbf{D}}_{H}^{n}, \\
& \hat{\mathbf{S}}_{H} \hat{\mathbf{P}}_{H}=\hat{\mathbf{P}}_{H} \hat{\mathbf{S}}_{H} \equiv 0, \quad \hat{\mathbf{S}}_{H} \hat{\mathbf{D}}_{H}=\hat{\mathbf{D}}_{H} \hat{\mathbf{S}}_{H} \equiv 0, \\
& \hat{\mathbf{P}}_{H} \hat{\mathbf{D}}_{H}=\hat{\mathbf{D}}_{H} \hat{\mathbf{P}}_{H}=\hat{\mathbf{D}}_{H} .
\end{aligned}
$$

Здесь оператор $\hat{\mathbf{D}}_{H}$ является собственным квазинильпотентным оператором, не имеющим невозмущенного аналога $\left(\hat{\mathbf{D}}_{H_{0}} \equiv 0\right)$. Таким образом, ряд Лорана для резольвенты оператора Лиувилля в окрестности $z=0$ принимает следующий общий вид [13]:

$$
\hat{\boldsymbol{R}}_{H}(z)=-\frac{1}{z} \hat{\mathbf{P}}_{H}+\sum_{n=0}^{\infty} z^{n} \hat{\mathbf{S}}_{H}^{n+1}-\sum_{n=2}^{\infty} z^{-n} \hat{\mathbf{D}}_{H}^{n-1}
$$


В то же время ряд для невозмущенной резольвенты состоит только из следующих членов:

$$
\hat{\boldsymbol{\mathcal { R }}}_{H_{0}}(z)=\sum_{n=0}^{+\infty} \hat{\mathbf{R}}_{H_{0}}^{(n)} z^{n-1}=-\frac{1}{z} \hat{\mathbf{P}}+\sum_{n=0}^{\infty} z^{n} \hat{\mathbf{S}}^{n+1}
$$

(напомним, что мы опускаем индекс $H_{0}$ ).

4.1.2. Канонические свойства резольвенты. Еще одно тождество, схожее с тождеством Гильберта, связывает резольвенту оператора Лиувилля и скобки Пуассона. Для любых $z_{1}, z_{2}, z_{3}$ вне спектра $\hat{\mathbf{L}}_{H}$ и функций $F, G$ выполняется следующее равенство:

$$
\begin{gathered}
\hat{\boldsymbol{\mathcal { R }}}_{H}\left(z_{1}\right)\left[\hat{\boldsymbol{\mathcal { R }}}_{H}\left(z_{2}\right) F, G\right]+\hat{\boldsymbol{\mathcal { R }}}_{H}\left(z_{1}\right)\left[F, \hat{\boldsymbol{\mathcal { R }}}_{H}\left(z_{3}\right) G\right]-\left[\hat{\boldsymbol{\mathcal { R }}}_{H}\left(z_{2}\right) F, \hat{\boldsymbol{\mathcal { R }}}_{H}\left(z_{3}\right) G\right]= \\
=\left(z_{1}-z_{2}-z_{3}\right) \hat{\boldsymbol{\mathcal { R }}}_{H}\left(z_{1}\right)\left[\hat{\boldsymbol{\mathcal { R }}}_{H}\left(z_{2}\right) F, \hat{\boldsymbol{\mathcal { R }}}_{H}\left(z_{3}\right) G\right] .
\end{gathered}
$$

Это еще одна формула интегрирования по частям. Она следует из применения единичного оператора $\hat{\boldsymbol{R}}_{H}\left(z_{1}\right)\left(\hat{\mathbf{L}}_{H}-z_{1}\right) \equiv 1$ к скобке Пуассона $\left[\hat{\boldsymbol{R}}_{H}\left(z_{2}\right) F, \hat{\boldsymbol{R}}_{H}\left(z_{3}\right) G\right]$ и тождества Якоби. Комплементарное тождество существует и для алгебраического произведения.

Мы будем использовать это каноническое тождество в операторной форме

$$
\begin{gathered}
\hat{\mathcal{R}}_{H}\left(z_{1}\right) \hat{\mathbf{L}}_{\hat{\mathcal{R}}_{H}\left(z_{2}\right) F}-\hat{\mathbf{L}}_{\hat{\mathcal{R}}_{H}\left(z_{2}\right) F} \hat{\boldsymbol{\mathcal { R }}}_{H}\left(z_{3}\right)+\hat{\boldsymbol{\mathcal { R }}}_{H}\left(z_{1}\right) \hat{\mathbf{L}}_{F} \hat{\boldsymbol{\mathcal { R }}}_{H}\left(z_{3}\right)= \\
=\left(z_{1}-z_{2}-z_{3}\right) \hat{\boldsymbol{\mathcal { R }}}_{H}\left(z_{1}\right) \hat{\mathbf{L}}_{\hat{\mathcal{R}}_{H}\left(z_{2}\right) F} \hat{\boldsymbol{\mathcal { R }}}_{H}\left(z_{3}\right) .
\end{gathered}
$$

Для невозмущенной резольвенты коэффициенты ряда Лорана в этом тождестве содержат все предыдущие канонические тождества (11)-(15). Например, коэффициент при $z_{1}^{0} z_{2}^{0} z_{3}^{0}$ есть тождество Фридрихса (13) и т. д. Интегрирование равенства (24) приводит к более сложным тождествам. Мы сделаем это в п. 4.3 для возмущенного случая.

4.2. Ряды Като. Резольвента возмущенной задачи может быть разложена в ряд Неймана:

$$
\begin{aligned}
\hat{\mathcal{R}}_{H_{0}+\alpha H_{j}}(z) & =\hat{\mathcal{R}}_{H_{0}}-\alpha \hat{\boldsymbol{\mathcal { R }}}_{H_{0}} \hat{\mathbf{L}}_{H_{j}} \hat{\boldsymbol{\mathcal { R }}}_{H_{0}}+\alpha^{2} \hat{\boldsymbol{\mathcal { R }}}_{H_{0}} \hat{\mathbf{L}}_{H_{j}} \hat{\boldsymbol{\mathcal { R }}}_{H_{0}} \hat{\mathbf{L}}_{H_{j}} \hat{\mathcal{R}}_{H_{0}}+\cdots= \\
& =\sum_{n=0}^{\infty}(-1)^{n} \alpha^{n} \hat{\boldsymbol{\mathcal { R }}}_{H_{0}}(z)\left(\hat{\mathbf{L}}_{H_{j}} \hat{\boldsymbol{\mathcal { R }}}_{H_{0}}(z)\right)^{n}
\end{aligned}
$$

Интегрирование по малому контуру приводит к разложениям Като [13] для возмущенного усредняющего оператора

$$
\begin{aligned}
\hat{\mathbf{P}}_{H} & =-\frac{1}{2 \pi i} \oint_{|z|=\epsilon} \hat{\boldsymbol{R}}_{H}(z) d z=-\frac{1}{2 \pi i} \sum_{n=0}^{\infty} \oint_{|z|=\epsilon}(-1)^{n} \alpha^{n} \hat{\boldsymbol{\mathcal { R }}}_{H_{0}}(z)\left(\hat{\mathbf{L}}_{H_{j}} \hat{\boldsymbol{\mathcal { R }}}_{H_{0}}(z)\right)^{n} d z= \\
& =-\frac{1}{2 \pi i} \sum_{n=0}^{\infty}(-1)^{n} \alpha^{n} \oint_{|z|=\epsilon}\left(\sum_{m=0}^{\infty} \hat{\mathbf{R}}_{H_{0}}^{(m)} z^{m-1}\right)\left(\hat{\mathbf{L}}_{H_{j}} \sum_{k=0}^{\infty} \hat{\mathbf{R}}_{H_{0}}^{(k)} z^{k-1}\right)^{n} d z,
\end{aligned}
$$

возмущенного интегрирующего оператора и возмущенного квазинильпотентного оператора

$$
\hat{\mathbf{S}}_{H}=\frac{1}{2 \pi i} \oint_{|z|=\epsilon} z^{-1} \hat{\mathcal{R}}_{H}(z) d z, \quad \hat{\mathbf{D}}_{H}=-\frac{1}{2 \pi i} \oint_{|z|=\epsilon} z \hat{\mathcal{R}}_{H}(z) d z
$$


Только коэффициенты порядка $z^{-1}$ в этих разложениях войдут в результат:

$$
\begin{aligned}
& \hat{\mathbf{P}}_{H}=\sum_{n=0}^{\infty}(-1)^{n+1} \alpha^{n}(\sum_{\substack{\sum p_{j}=\mathbf{n} \\
p_{j} \geqslant 0}} \hat{\mathbf{R}}_{H_{0}}^{\left(p_{n+1}\right)} \underbrace{\hat{\mathbf{L}}_{H_{j}} \hat{\mathbf{R}}_{H_{0}}^{\left(p_{n}\right)} \ldots \hat{\mathbf{R}}_{H_{0}}^{\left(p_{2}\right)} \hat{\mathbf{L}}_{H_{j}}}_{n \text { раз }} \hat{\mathbf{R}}_{H_{0}}^{\left(p_{1}\right)}), \\
& \hat{\mathbf{S}}_{H}=\sum_{n=0}^{\infty}(-1)^{n} \quad \alpha^{n}(\sum_{\substack{\sum p_{j}=\mathbf{n}+\mathbf{1} \\
p_{j} \geqslant 0}} \hat{\mathbf{R}}_{H_{0}}^{\left(p_{n+1}\right)} \underbrace{\hat{\mathbf{L}}_{H_{j}} \hat{\mathbf{R}}_{H_{0}}^{\left(p_{n}\right)} \ldots \hat{\mathbf{R}}_{H_{0}}^{\left(p_{2}\right)} \hat{\mathbf{L}}_{H_{j}}}_{n \text { раз }} \hat{\mathbf{R}}_{H_{0}}^{\left(p_{1}\right)}), \\
& \hat{\mathbf{D}}_{H}=\sum_{n=1}^{\infty}(-1)^{n+1} \alpha^{n}(\sum_{\substack{\sum p_{j}=\mathbf{n}-1 \\
p_{j} \geqslant 0}} \hat{\mathbf{R}}_{H_{0}}^{\left(p_{n+1}\right)} \underbrace{\hat{\mathbf{L}}_{H_{j}} \hat{\mathbf{R}}_{H_{0}}^{\left(p_{n}\right)} \ldots \hat{\mathbf{R}}_{H_{0}}^{\left(p_{2}\right)} \hat{\mathbf{L}}_{H_{j}}}_{n \text { раз }} \hat{\mathbf{R}}_{H_{0}}^{\left(p_{1}\right)}) .
\end{aligned}
$$

В каждом порядке этих выражений суммирование производится по всем возможным расстановкам $n$ (или $n+1$, или $n-1$ ) операторов $\hat{\mathbf{S}}_{H_{0}}$ по $n+1$ позициям. Выражение порядка $\alpha^{n}$ для $\hat{\mathbf{P}}_{H}$ состоит из $C_{2 n}^{n}$ слагаемых, а для $\hat{\mathbf{S}}_{H}-$ из $C_{2 n+1}^{n}$ слагаемых. Приведем первые два порядка для возмущенного интегрирующего оператора:

$$
\begin{aligned}
\hat{\mathbf{S}}_{H}= & \hat{\mathbf{S}}-\alpha\left(\hat{\mathbf{S}} \hat{\mathbf{L}}_{H_{j}} \hat{\mathbf{S}}-\hat{\mathbf{S}}^{2} \hat{\mathbf{L}}_{H_{j}} \hat{\mathbf{P}}-\hat{\mathbf{P}} \hat{\mathbf{L}}_{H_{j}} \hat{\mathbf{S}}^{2}\right)+\alpha^{2}\left(\hat{\mathbf{S}} \hat{\mathbf{L}}_{H_{j}} \hat{\mathbf{S}} \hat{\mathbf{L}}_{H_{j}} \hat{\mathbf{S}}-\hat{\mathbf{S}}^{2} \hat{\mathbf{L}}_{H_{j}} \hat{\mathbf{S}} \hat{\mathbf{L}}_{H_{j}} \hat{\mathbf{P}}-\right. \\
& -\hat{\mathbf{S}}^{2} \hat{\mathbf{L}}_{H_{j}} \hat{\mathbf{P}} \hat{\mathbf{L}}_{H_{j}} \hat{\mathbf{S}}-\hat{\mathbf{P}} \hat{\mathbf{L}}_{H_{j}} \hat{\mathbf{S}}^{2} \hat{\mathbf{L}}_{H_{j}} \hat{\mathbf{S}}-\hat{\mathbf{S}} \hat{\mathbf{L}}_{H_{j}} \hat{\mathbf{S}}^{2} \hat{\mathbf{L}}_{H_{j}} \hat{\mathbf{P}}-\hat{\mathbf{S}} \hat{\mathbf{L}}_{H_{j}} \hat{\mathbf{P}} \hat{\mathbf{L}}_{H_{j}} \hat{\mathbf{S}}^{2}-\hat{\mathbf{P}} \hat{\mathbf{L}}_{H_{j}} \hat{\mathbf{S}} \hat{\mathbf{L}}_{H_{j}} \hat{\mathbf{S}}^{2}+ \\
& \left.+\hat{\mathbf{P}} \hat{\mathbf{L}}_{H_{j}} \hat{\mathbf{P}} \hat{\mathbf{L}}_{H_{j}} \hat{\mathbf{S}}^{3}+\hat{\mathbf{P}} \hat{\mathbf{L}}_{H_{j}} \hat{\mathbf{S}}^{3} \hat{\mathbf{L}}_{H_{j}} \hat{\mathbf{P}}+\hat{\mathbf{S}}^{3} \hat{\mathbf{L}}_{H_{j}} \hat{\mathbf{P}} \hat{\mathbf{L}}_{H_{j}} \hat{\mathbf{P}}\right)+O\left(\alpha^{3}\right),
\end{aligned}
$$

возмущенного проектора:

$$
\begin{aligned}
\hat{\mathbf{P}}_{H}= & \hat{\mathbf{P}}-\alpha\left(\hat{\mathbf{P}} \hat{\mathbf{L}}_{H_{j}} \hat{\mathbf{S}}+\hat{\mathbf{S}} \hat{\mathbf{L}}_{H_{j}} \hat{\mathbf{P}}\right)+\alpha^{2}\left(\hat{\mathbf{P}} \hat{\mathbf{L}}_{H_{j}} \hat{\mathbf{S}} \hat{\mathbf{L}}_{H_{j}} \hat{\mathbf{S}}+\hat{\mathbf{S}} \hat{\mathbf{L}}_{H_{j}} \hat{\mathbf{P}} \hat{\mathbf{L}}_{H_{j}} \hat{\mathbf{S}}+\right. \\
& \left.+\hat{\mathbf{S}} \hat{\mathbf{L}}_{H_{j}} \hat{\mathbf{S}} \hat{\mathbf{L}}_{H_{j}} \hat{\mathbf{P}}-\hat{\mathbf{P}} \hat{\mathbf{L}}_{H_{j}} \hat{\mathbf{P}} \hat{\mathbf{L}}_{H_{j}} \hat{\mathbf{S}}^{2}-\hat{\mathbf{P}} \hat{\mathbf{L}}_{H_{j}} \hat{\mathbf{S}}^{2} \hat{\mathbf{L}}_{H_{j}} \hat{\mathbf{P}}-\hat{\mathbf{S}}^{2} \hat{\mathbf{L}}_{H_{j}} \hat{\mathbf{P}} \hat{\mathbf{L}}_{H_{j}} \hat{\mathbf{P}}\right)+O\left(\alpha^{3}\right),
\end{aligned}
$$

и, наконец, для возмущенного квазинильпотентного оператора:

$$
\hat{\mathbf{D}}_{H}=\alpha \hat{\mathbf{P}} \hat{\mathbf{L}}_{H_{j}} \hat{\mathbf{P}}-\alpha^{2}\left(\hat{\mathbf{P}} \hat{\mathbf{L}}_{H_{j}} \hat{\mathbf{P}} \hat{\mathbf{L}}_{H_{j}} \hat{\mathbf{S}}+\hat{\mathbf{P}} \hat{\mathbf{L}}_{H_{j}} \hat{\mathbf{S}} \hat{\mathbf{L}}_{H_{j}} \hat{\mathbf{P}}+\hat{\mathbf{S}} \hat{\mathbf{L}}_{H_{j}} \hat{\mathbf{P}} \hat{\mathbf{L}}_{H_{j}} \hat{\mathbf{P}}\right)+O\left(\alpha^{3}\right) .
$$

Свойства невозмущенных операторов распространяются на их аналитические продолжения следующим образом:

$$
\hat{\mathbf{P}}_{H} H=H, \quad \hat{\mathbf{S}}_{H} \hat{\mathbf{L}}_{H}=1-\hat{\mathbf{P}}_{H}, \quad \hat{\mathbf{L}}_{H} \hat{\mathbf{P}}_{H}=\hat{\mathbf{P}}_{H} \hat{\mathbf{L}}_{H}=\hat{\mathbf{D}}_{H}, \quad \ldots .
$$

Детали можно найти в приложении А и демонстрации "perturbed_operators".

Квантово-механическая теория возмущений использует ряд для $\hat{\mathbf{P}}_{H}$ при нахождении собственных значений возмущенного гамильтониана [2]. В классической механике нет прямого аналога этой процедуры. Во избежание недоразумений следует отметить, что $\hat{\mathbf{P}}_{H} F$ не будет интегралом движения возмущенного гамильтониана, так как $\hat{\mathbf{L}}_{H} \hat{\mathbf{P}}_{H}=\hat{\mathbf{D}}_{H} \neq 0$ в общем случае.

На самом деле возмущенный проектор $\hat{\mathbf{P}}_{H}$ проецирует на аналитическое продолжение алгебры интегралов невозмущенного гамильтониана. Это пространство не совпадает, вообще говоря, с алгеброй интегралов возмущенной системы, так как часть интегралов разрушается. Другими словами, нулевое собственное значение оператора Лиувилля может расщепляться возмущением. 
4.3. Связь с разложениями Пуанкаре-Линдштедта. Чтобы установить соответствие между разложениями Като и стандартной классической теорией возмущений, мы продемонстрируем, что проекционные операторы $\hat{\mathbf{P}}_{H}$ и $\hat{\mathbf{P}}_{H_{0}}$ канонически связаны. Именно это является точкой объединения двух столь различных формализмов.

Действительно, если мы построим каноническое преобразование

$$
\widetilde{\mathbf{x}}=\exp _{\mathrm{D}}\left(\alpha \hat{\mathbf{L}}_{G}\right) \mathbf{x}, \quad \widetilde{H}=\exp _{\mathrm{D}}^{-1}\left(\alpha \hat{\mathbf{L}}_{G}\right) H,
$$

связывающее проекторы

$$
\hat{\mathbf{P}}_{H}=\exp _{\mathrm{D}}\left(\alpha \hat{\mathbf{L}}_{G}\right) \hat{\mathbf{P}}_{H_{0}} \exp _{\mathrm{D}}^{-1}\left(\alpha \hat{\mathbf{L}}_{G}\right)
$$

то из равенства $\hat{\mathbf{P}}_{H} H=H$ будет следовать, что преобразованный гамильтониан будет интегралом движения невозмущенной системы:

$$
\hat{\mathbf{P}}_{H_{0}} \widetilde{H}=\hat{\mathbf{P}}_{H_{0}} \exp _{\mathrm{D}}^{-1}\left(\alpha \hat{\mathbf{L}}_{G}\right) H=\exp _{\mathrm{D}}^{-1}\left(\alpha \hat{\mathbf{L}}_{G}\right) \hat{\mathbf{P}}_{H} H=\exp _{\mathrm{D}}^{-1}\left(\alpha \hat{\mathbf{L}}_{G}\right) H=\widetilde{H} .
$$

Следовательно, такое преобразование явным образом реализует программу теории возмущений Пуанкаре--Линдштедта.

Для того чтобы найти это преобразование, рассмотрим производную резольвенты оператора Лиувилля по возмущению:

$$
\frac{\partial}{\partial \alpha} \hat{\mathcal{R}}_{H}(z)=-\hat{\mathcal{R}}_{H}(z) \hat{\mathbf{L}}_{H_{j}} \hat{\boldsymbol{\mathcal { R }}}_{H}(z)
$$

Подстановка $z_{1}=z_{3}=z$ и $F=H_{j}$ в резольвентное каноническое тождество (24) дает

$$
\begin{aligned}
\hat{\mathcal{R}}_{H}(z) \hat{\mathbf{L}}_{H_{j}} \hat{\boldsymbol{\mathcal { R }}}_{H}(z)= & \hat{\mathbf{L}}_{\hat{\mathcal{R}}_{H}\left(z_{2}\right) H_{j}} \hat{\boldsymbol{\mathcal { R }}}_{H}(z)-\hat{\mathcal{R}}_{H}(z) \hat{\mathbf{L}}_{\hat{\mathcal{R}}_{H}\left(z_{2}\right) H_{j}}- \\
& -z_{2} \hat{\boldsymbol{\mathcal { R }}}_{H}(z) \hat{\mathbf{L}}_{\hat{\mathcal{R}}_{H}\left(z_{2}\right) H_{j}} \hat{\boldsymbol{\mathcal { R }}}_{H}(z) .
\end{aligned}
$$

Рассмотрим коэффициент ряда Лорана при $z_{2}^{0}$ в этом выражении:

$$
\frac{\partial}{\partial \alpha} \hat{\boldsymbol{\mathcal { R }}}_{H}(z)=\hat{\boldsymbol{\mathcal { R }}}_{H}(z) \hat{\mathbf{L}}_{\hat{\mathbf{S}}_{H} H_{j}}-\hat{\mathbf{L}}_{\hat{\mathbf{s}}_{H} H_{j}} \hat{\boldsymbol{\mathcal { R }}}_{H}(z)-\hat{\boldsymbol{\mathcal { R }}}_{H}(z) \hat{\mathbf{L}}_{\hat{\mathbf{P}}_{H} H_{j}} \hat{\boldsymbol{\mathcal { R }}}_{H}(z) .
$$

Поступая аналогично для коэффициентов в $(27)$ при $z_{2}^{-n}(n \geqslant 1)$, получим

$$
\begin{aligned}
& \hat{\mathcal{R}}_{H}(z) \hat{\mathbf{L}}_{\hat{\mathbf{P}}_{H} H_{j}}=\hat{\mathbf{L}}_{\hat{\mathbf{P}}_{H} H_{j}} \hat{\boldsymbol{\mathcal { R }}}_{H}(z)-\hat{\mathcal{R}}_{H}(z) \hat{\mathbf{L}}_{\hat{\mathbf{D}}_{H} H_{j}} \hat{\boldsymbol{\mathcal { R }}}_{H}(z), \\
& \hat{\boldsymbol{\mathcal { R }}}_{H}(z) \hat{\mathbf{L}}_{\hat{\mathbf{D}}_{H}^{n} H_{j}}=\hat{\mathbf{L}}_{\hat{\mathbf{D}}_{H}^{n} H_{j}} \hat{\boldsymbol{\mathcal { R }}}_{H}(z)-\hat{\mathcal{R}}_{H}(z) \hat{\mathbf{L}}_{\hat{\mathbf{D}}_{H}^{n+1} H_{j}} \hat{\boldsymbol{\mathcal { R }}}_{H}(z) .
\end{aligned}
$$

Это позволяет переписать выражение для производной резольвенты в виде

$$
\begin{aligned}
\frac{\partial}{\partial \alpha} \hat{\boldsymbol{R}}_{H}(z)= & \hat{\mathcal{R}}_{H}(z) \hat{\mathbf{L}}_{\hat{\mathbf{s}}_{H} H_{j}}-\hat{\mathbf{L}}_{\hat{\mathbf{s}}_{H} H_{j}} \hat{\mathcal{R}}_{H}(z)- \\
& -\hat{\mathbf{L}}_{\hat{\mathbf{P}}_{H} H_{j}} \hat{\boldsymbol{\mathcal { R }}}_{H}(z)^{2}+\hat{\mathbf{L}}_{\hat{\mathbf{D}}_{H} H_{j}} \hat{\mathcal{R}}_{H}(z)^{3}-\hat{\mathbf{L}}_{\hat{\mathbf{D}}_{H}^{2} H_{j}} \hat{\mathcal{R}}_{H}(z)^{4}+\cdots
\end{aligned}
$$

Это степенной ряд, так как $\hat{\mathbf{D}}_{H}^{n}=O\left(\alpha^{n}\right)$. 
Из тождества Гильберта следует, что

$$
\frac{\partial^{n}}{\partial z^{n}} \hat{\mathcal{R}}_{H}(z)=n ! \hat{\mathcal{R}}_{H}^{n+1}(z)
$$

Окончательно получаем

$$
\begin{aligned}
& \frac{\partial}{\partial \alpha} \hat{\boldsymbol{R}}_{H}(z)=\hat{\mathcal{R}}_{H}(z) \hat{\mathbf{L}}_{\hat{\mathbf{s}}_{H} H_{j}}-\hat{\mathbf{L}}_{\hat{\mathbf{s}}_{H} H_{j}} \hat{\mathcal{R}}_{H}(z)-\hat{\mathbf{L}}_{\hat{\mathbf{P}}_{H} H_{j}} \frac{\partial \hat{\mathcal{R}}_{H}(z)}{\partial z}+ \\
& +\frac{1}{2} \hat{\mathbf{L}}_{\hat{\mathbf{D}}_{H} H_{j}} \frac{\partial^{2} \hat{\boldsymbol{\mathcal { R }}}_{H}(z)}{\partial z^{2}}-\frac{1}{6} \hat{\mathbf{L}}_{\hat{\mathbf{D}}_{H}^{2} H_{j}} \frac{\partial^{3} \hat{\boldsymbol{\mathcal { R }}}_{H}(z)}{\partial z^{3}}+\cdots .
\end{aligned}
$$

Таким образом, изменение резольвенты оператора Лиувилля под действием возмущения $\alpha H_{j}$ может быть представлено как сумма канонического преобразования с генератором - $\hat{\mathbf{S}}_{H} H_{j}$ и трансформации резольвенты как функции комплексного переменного $z$.

Производная проектора $\partial \hat{\mathbf{P}}_{H} / \partial \alpha$ может быть получена как вычет предыдущего тождества в $z=0$. В нашем случае чисто дискретного спектра резольвента мероморфна, поэтому вычет любой ее производной по $z$ тождественно равен нулю. Следовательно, проектор $\hat{\mathbf{P}}_{H}$ преобразуется канонически под действием возмущения:

$$
\frac{\partial}{\partial \alpha} \hat{\mathbf{P}}_{H}=\hat{\mathbf{P}}_{H} \hat{\mathbf{L}}_{\hat{\mathbf{s}}_{H} H_{j}}-\hat{\mathbf{L}}_{\hat{\mathbf{s}}_{H} H_{j}} \hat{\mathbf{P}}_{H},
$$

и проекторы связаны преобразованием Ли-Депри:

$$
\hat{\mathbf{P}}_{H}=\exp _{\mathrm{D}}\left(\alpha \hat{\mathbf{L}}_{-\hat{\mathbf{s}}_{H} H_{j}}\right) \hat{\mathbf{P}}_{H_{0}} \exp _{\mathrm{D}}^{-1}\left(\alpha \hat{\mathbf{L}}_{-\hat{\mathbf{s}}_{H} H_{j}}\right) .
$$

Таким образом, мы показали, что каноническое преобразование с генератором

$$
G=-\hat{\mathbf{S}}_{H} H_{j}=\sum_{n=0}^{\infty}(-1)^{n+1} \alpha^{n}(\sum_{\substack{\sum p_{j}=\mathbf{n}+\mathbf{1}, p_{j} \geqslant 0}} \hat{\mathbf{R}}_{H_{0}}^{\left(p_{n+1}\right)} \underbrace{\hat{\mathbf{L}}_{H_{j}} \hat{\mathbf{R}}_{H_{0}}^{\left(p_{n}\right)} \ldots \hat{\mathbf{R}}_{H_{0}}^{\left(p_{2}\right)} \hat{\mathbf{L}}_{H_{j}}}_{n \text { раз }} \hat{\mathbf{R}}_{H_{0}}^{\left(p_{1}\right)} H_{j})
$$

формально нормализует гамильтониан во всех порядках по $\alpha$.

4.4. Общая форма генератора. Зная, что $\hat{\mathbf{P}}_{H_{0}}$ и $\hat{\mathbf{P}}_{H}$ канонически связаны и это преобразование нормализует гамильтониан, можно переформулировать программу теории возмущений Пуанкаре-Линдштедта в виде конструирования канонического преобразования, связывающего невозмущенный и возмущенный усредняющие операторы.

Выясним теперь, какова общая форма генератора такого преобразования. Из (26) следует, что $\hat{\mathbf{P}}_{H}$ удовлетворяет операторному дифференциальному уравнению

$$
\frac{\partial}{\partial \alpha} \hat{\mathbf{P}}_{H}=\hat{\mathbf{L}}_{G} \hat{\mathbf{P}}_{H}-\hat{\mathbf{P}}_{H} \hat{\mathbf{L}}_{G}
$$

Применение этого выражения к гамильтониану $H$ дает

$$
\left(\frac{\partial}{\partial \alpha} \hat{\mathbf{P}}_{H}\right) H=\hat{\mathbf{L}}_{G} \hat{\mathbf{P}}_{H} H-\hat{\mathbf{P}}_{H} \hat{\mathbf{L}}_{G} H .
$$

5 Теоретическая и математическая физика, т. 182, № 3, 2015 г. 
Так как

$$
\hat{\mathbf{P}}_{H} H=H, \quad \frac{\partial}{\partial \alpha}\left(\hat{\mathbf{P}}_{H} H\right)=\left(\frac{\partial}{\partial \alpha} \hat{\mathbf{P}}_{H}\right) H+\hat{\mathbf{P}}_{H} \frac{\partial}{\partial \alpha} H, \quad \frac{\partial}{\partial \alpha} H=H_{j},
$$

то предыдущее выражение принимает вид

$$
\left(1-\hat{\mathbf{P}}_{H}\right) \hat{\mathbf{L}}_{H} G=-\left(1-\hat{\mathbf{P}}_{H}\right) H_{j} .
$$

Для решения этого уравнения достаточно применить оператор $\hat{\mathbf{S}}_{H}$. Следовательно, общая форма генератора преобразования, связывающего проекторы, имеет вид

$$
G=-\hat{\mathbf{S}}_{H} H_{j}+\hat{\mathbf{P}}_{H} F,
$$

где $F(\mathbf{x}, \alpha)$ - любая аналитическая функция. Эта формула - главный результат настоящей работы. Она дает нерекуррентное выражение для генератора Депри нормализующего преобразования Пуанкаре-Линдштедта и определяет его возможную неоднозначность.

Выбор функции $F$ определяет стиль нормализации. Естественно выбрать $F \equiv 0$ или $\hat{\mathbf{P}}_{H} G=0$. Это не эквивалентно "несекулярному" стилю $\hat{\mathbf{P}}_{H_{0}} G_{D}=0$, традиционно используемому в теории возмущений классической механики. Так как $\hat{\mathbf{L}}_{\hat{\mathbf{P}}_{H} F} \hat{\mathbf{P}}_{H}=\hat{\mathbf{P}}_{H} \hat{\mathbf{L}}_{\hat{\mathbf{P}}_{H} F}$, сам проектор $\hat{\mathbf{P}}_{H}$ не чувствителен к стилю нормализации.

Мы можем заключить, что генераторы нормализующих преобразований могут различаться на функиию, принадлежашую аналитическому продолжсению алгебры интегралов невозмущенной системы. Иллюстрацию к этому можно найти в демонстрации "styles".

Заметим, что квантово-механическая теория возмущений Като использует другие формулы для унитарного преобразования, связывающего невозмущенный и возмущенный проекторы [13]. Но оригинальный генератор Като и рациональное выражение, полученное Секефальви и Надем (см. [13]), не определяют каноническое преобразование в классической механике.

4.5. Первые порядки. Сравним выражения для генератора

$$
\begin{aligned}
G= & -\hat{\mathbf{S}} H_{j}+\alpha\left(\hat{\mathbf{S}} \hat{\mathbf{L}} \hat{\mathbf{S}} H_{j}-\hat{\mathbf{S}}^{2} \hat{\mathbf{L}} \hat{\mathbf{P}} H_{j}\right)-\alpha^{2}\left(\hat{\mathbf{S}} \hat{\mathbf{L}} \hat{\mathbf{L}} \hat{\mathbf{S}} H_{j}-\hat{\mathbf{S}} \hat{\mathbf{L}} \hat{S}^{2} \hat{\mathbf{L}} \hat{\mathbf{P}} H_{j}-\right. \\
& -\hat{\mathbf{S}}^{2} \hat{\mathbf{L}} \hat{\mathbf{S}} \hat{\mathbf{P}} H_{j}-\hat{\mathbf{S}}^{2} \hat{\mathbf{L}} \hat{\mathbf{P}} \hat{\mathbf{L}} H_{j}-\hat{\mathbf{P}} \hat{\mathbf{L}} \hat{\mathbf{L}} \hat{\mathbf{S}}^{2} H_{j}-\hat{\mathbf{P}} \hat{\mathbf{L}} \hat{\mathbf{S}}^{2} \hat{\mathbf{L}} \hat{\mathbf{S}} H_{j}+ \\
& \left.+\hat{\mathbf{S}}^{3} \hat{\mathbf{L}} \hat{\mathbf{P}} \hat{\mathbf{L}} \hat{\mathbf{P}} H_{j}+\hat{\mathbf{P}} \hat{\mathbf{L}} \hat{\mathbf{S}}^{3} \hat{\mathbf{L}} \hat{\mathbf{P}} H_{j}+\hat{\mathbf{P}} \hat{\mathbf{L}} \hat{\mathbf{P}} \hat{\mathbf{S}}^{3} H_{j}\right)+O\left(\alpha^{3}\right)
\end{aligned}
$$

и нормализованного гамильтониана

$$
\begin{aligned}
\widetilde{H}= & H_{0}+\alpha \hat{\mathbf{P}} H_{j}-\frac{\alpha^{2}}{2} \hat{\mathbf{P}} \hat{\mathbf{L}} \hat{\mathbf{S}} H_{j}+\alpha^{3}\left(\frac{1}{3} \hat{\mathbf{P}} \hat{\mathbf{L}} \hat{\mathbf{L}} \hat{\mathbf{S}} H_{j}-\frac{1}{6} \hat{\mathbf{P}} \hat{\mathbf{L}} \hat{\mathbf{S}}^{2} \hat{\mathbf{L}} \hat{\mathbf{P}} H_{j}\right)+ \\
& +\alpha^{4}\left(\frac{1}{6} \hat{\mathbf{P}} \hat{\mathbf{L}} \hat{\mathbf{S}} \hat{\mathbf{S}}^{2} \hat{\mathbf{L}} \hat{\mathbf{P}} H_{j}-\frac{1}{4} \hat{\mathbf{P}} \hat{\mathbf{L}} \hat{\mathbf{L}} \hat{\mathbf{S}} \hat{\mathbf{L}} H_{j}+\frac{1}{12} \hat{\mathbf{P}} \hat{\mathbf{L}} \hat{\mathbf{S}}^{2} \hat{\mathbf{L}} \hat{\mathbf{S}} \hat{\mathbf{L}} H_{j}+\right. \\
& +\frac{1}{8} \hat{\mathbf{P}} \hat{\mathbf{L S}}^{2} \hat{\mathbf{L}} \hat{\mathbf{P}} \hat{\mathbf{S}} H_{j}+\frac{1}{4} \hat{\mathbf{P}} \hat{\mathbf{L}} \hat{\mathbf{P}} \hat{\mathbf{S}}^{2} \hat{\mathbf{L}} \hat{\mathbf{S}} H_{j}+\frac{1}{4} \hat{\mathbf{P}} \hat{\mathbf{L}} \hat{\mathbf{P}} \hat{\mathbf{S}} \hat{\mathbf{L}} \hat{\mathbf{S}}^{2} H_{j}- \\
& \left.-\frac{1}{6} \hat{\mathbf{P}} \hat{\mathbf{L}} \hat{\mathbf{P}} \hat{\mathbf{S}}^{3} \hat{\mathbf{L}} \hat{\mathbf{P}} H_{j}-\frac{1}{4} \hat{\mathbf{P}} \hat{\mathbf{L}} \hat{\mathbf{P}} \hat{\mathbf{P}} \hat{\mathbf{L}} \hat{\mathbf{S}}^{3} H_{j}\right)+O\left(\alpha^{5}\right)
\end{aligned}
$$


с рядами Депри (16). Здесь для краткости мы обозначили $\hat{\mathbf{L}}=\hat{\mathbf{L}}_{H_{j}}$ и использовали тождества $\hat{\mathbf{P}} \hat{\mathbf{L}}_{H_{j}} \hat{\mathbf{S}}^{2} H_{j} \equiv 0$ и $\hat{\mathbf{P}} \hat{\mathbf{L}} \hat{\mathbf{P}} H_{j} \equiv 0$.

До третьего порядка выражения (16) и (33) очень близки и различаются только секулярными членами в генераторе. Эта разница связана с "естественным" стилем нормализации $\hat{\mathbf{P}}_{H} G=0$. В высших порядках эти различия распространяются и на несекулярные члены. Благодаря этим дополнительным членам наши выражения для генератора и нормализованного гамильтониана становятся линейными и систематизированными. На самом деле благодаря каноническим тождествам существует много эквивалентных выражений для генератора и гамильтониана.

Большая формула для гамильтониана, нормализованного до $O\left(\alpha^{8}\right)$, приводится в демонстрации "normalized_hamiltonian7". Она состоит из 528 членов. Соответствующий генератор состоит из 2353 членов.

ПримеР 1. УРАВНЕНИЕ ДЮФФИНГА. Гамильтониан ангармонического осциллятора с нелинейностью четвертого порядка имеет вид

$$
H=\frac{1}{2}\left(p^{2}+q^{2}\right)+\frac{\alpha}{4} q^{4}=J+\alpha J^{2} \cos ^{4} \phi .
$$

Нормализующий генератор в представлениях Биркгофа и “действие-угол"

$$
\begin{aligned}
G & =\frac{p q}{32}\left(3 p^{2}+5 q^{2}\right)-\alpha \frac{p q}{384}\left(39 p^{4}+104 p^{2} q^{2}+57 q^{4}\right)+O\left(\alpha^{2}\right)= \\
& =-\frac{J^{2}}{32}(8 \sin 2 \phi+\sin 4 \phi)+\frac{\alpha J^{3}}{192}(99 \sin 2 \phi+9 \sin 4 \phi-\sin 6 \phi)+O\left(\alpha^{2}\right),
\end{aligned}
$$

а нормализованный гамильтониан имеет следующий вид:

$$
\begin{aligned}
\widetilde{H} & =\frac{1}{2}\left(p^{2}+q^{2}\right)+\frac{3 \alpha}{32}\left(p^{2}+q^{2}\right)^{2}-\frac{17 \alpha^{2}}{512}\left(p^{2}+q^{2}\right)^{3}+\frac{375 \alpha^{3}}{16384}\left(p^{2}+q^{2}\right)^{4}+O\left(\alpha^{4}\right)= \\
& =J+\frac{3}{8} \alpha J^{2}-\frac{17}{64} \alpha^{2} J^{3}+\frac{375}{1024} \alpha^{3} J^{4}+O\left(\alpha^{4}\right) .
\end{aligned}
$$

Это типичная структура пертурбативного ряда для нерезонансных систем. Следующие члены можно найти в демонстрации "anharmonic". Благодаря уникальности нерезонансной нормальной формы [23] она совпадает с классическим рядом Депри.

\section{5. МНОГОМЕРНЫЕ СИСТЕМЫ}

5.1. Усеченные ряды. Распространение предыдущих построений на многомерный случай вызывает трудности. Спектр оператора Лиувилля для многочастотной динамической системы является объединением счетного количества аддитивных групп [14]. Типичным является то, что сингулярности резольвенты многомерного оператора Лиувилля не могут быть отделены от начала координат. Это проявление классической проблемы малых знаменателей. Резольвента более не голоморфна [18], и, следовательно, мы не можем непосредственно полагаться на ряды Като (25) и вычеты. 
Однако наши компьютерные вычисления подтверждают справедливость выражения (30) для генератора в первых порядках и в многомерном случае. Ключевой здесь является каноническая связь между возмущенным и невозмущенным проекторами. Используя демонстрацию "phdot", мы проверили ее прямыми вычислениями вплоть до $\alpha^{10}$. Упрощение почти трех миллионов членов заняло неделю компьютерного времени.

Следовательно, мы можем предположить, что формула (30) остается (асимптотически) верной и в многомерном случае. Но нам нужно другое доказательство, использующее канонические тождества (11)-(14) и не опирающееся на резольвенту оператора Лиувилля.

Такое доказательство приведено в приложениях А, Б. Оно следует идеям предыдущего раздела, но использует конечные, усеченные в порядке $N$ операторные суммы. В приложении А обсуждаются свойства усеченных возмущенных операторов $\hat{\mathbf{P}}_{H}^{[N]}, \hat{\mathbf{S}}_{H}^{[N]}$ и $\hat{\mathbf{D}}_{H}^{[N]}$, а в приложении Б мы демонстрируем, что для любого $N \in \mathbb{N}$, $N \geqslant 2$, возмущенный проектор преобразуется канонически:

$$
\left(\frac{\partial}{\partial \alpha} \hat{\mathbf{P}}_{H}\right)^{[N]}=\hat{\mathbf{L}}_{-\hat{\mathbf{S}}_{H}^{[N]} H_{j}} \hat{\mathbf{P}}_{H}^{[N]}-\hat{\mathbf{P}}_{H}^{[N]} \hat{\mathbf{L}}_{-\hat{\mathbf{S}}_{H}^{[N]} H_{j}}+O\left(\alpha^{N+1}\right) .
$$

Используя экспоненты Депри, мы можем заключить, что преобразование ЛиДепри с генератором $G^{[N]}=-\hat{\mathbf{S}}_{H}^{[N]} H_{j}$ нормализует гамильтониан до порядка $N$ :

$$
\begin{aligned}
\widetilde{H}^{[N]} & =\exp _{\mathrm{D}}^{-1}\left(\alpha \hat{\mathbf{L}}_{-\hat{\mathbf{S}}_{H}^{[N]} H_{j}}\right) H+O\left(\alpha^{N+1}\right)=\exp _{\mathrm{D}}^{-1}\left(\alpha \hat{\mathbf{L}}_{-\hat{\mathbf{S}}_{H}^{[N]} H_{j}}\right) \hat{\mathbf{P}}_{H}^{[N]} H+O\left(\alpha^{N+1}\right)= \\
& =\hat{\mathbf{P}}_{H_{0}} \exp _{\mathrm{D}}^{-1}\left(\alpha \hat{\mathbf{L}}_{-\hat{\mathbf{S}}_{H}^{[N]} H_{j}}\right) H+O\left(\alpha^{N+1}\right)=\hat{\mathbf{P}}_{H_{0}} \widetilde{H}^{[N]}+O\left(\alpha^{N+1}\right) .
\end{aligned}
$$

Это устанавливает формально асимптотический характер рядов.

Малые знаменатели также затрагивают и невозмущенные операторы $\hat{\mathbf{P}}_{H_{0}}$ и $\hat{\mathbf{S}}_{H_{0}}$, определяемые выражениями (8). Эта классическая проблема известна в небесной механике с 1884 г. (Г. Брунс). В фундаментальных работах Колмогорова [15] и Арнольда [16] всеобъемлюще исследованы аналитические свойства операции усреднения и решения гомологического уравнения. Для невырожденной многомерной системы общего положения эти операторы аналитичны для почти всех частот, кроме множества лебеговой меры нуль. Этого достаточно для наших формальных построений. Следует отметить, что данная проблема не затрагивает приложений, в которых возмущение представлено конечным рядом Фурье.

5.2. Неоднозначности преобразованного гамильтониана. Аналогично (31) общей формой усеченного генератора преобразования Пуанкаре-Линдштедта является

$$
G^{[N]}=-\hat{\mathbf{S}}_{H}^{[N]} H_{j}+\hat{\mathbf{P}}_{H}^{[N]} F,
$$

где $F(\mathbf{x}, \alpha)$ определяет стиль нормализации. Теперь мы можем обсудить его эффект.

Для краткости здесь и далее мы будем использовать формально-аналитические выражения, как и выше. Но мы будем всегда подразумевать, что для математической корректности эти выражения должны быть превращены в усеченные суммы, а все равенства будут выполняться с точностью до $O\left(\alpha^{N+1}\right)$. 
Следуя [23], рассмотрим два гамильтониана, нормализованных в различных стилях: $\widetilde{H}_{1}=\exp _{\mathrm{D}}^{-1}\left(\alpha \hat{\mathbf{L}}_{G_{1}}\right) H$ и $\widetilde{H}_{2}=\exp _{\mathrm{D}}^{-1}\left(\alpha \hat{\mathbf{L}}_{G_{2}}\right) H$. Эти величины связаны преобразованием

$$
\widetilde{H}_{2}=\exp _{\mathrm{D}}^{-1}\left(\alpha \hat{\mathbf{L}}_{G_{2}}\right) \exp _{\mathrm{D}}\left(\alpha \hat{\mathbf{L}}_{G_{1}}\right) \widetilde{H}_{1}=\widehat{\mathbf{U}}_{21} \widetilde{H}_{1}
$$

Очевидно, преобразование $\widehat{\mathbf{U}}_{21}$ каноническое. Найдем его генератор:

$$
\begin{aligned}
\frac{\partial}{\partial \alpha} \widehat{\mathbf{U}}_{21} & =\exp _{\mathrm{D}}^{-1}\left(\alpha \hat{\mathbf{L}}_{G_{2}}\right)\left(\hat{\mathbf{L}}_{G_{1}}-\hat{\mathbf{L}}_{G_{2}}\right) \exp _{\mathrm{D}}\left(\alpha \hat{\mathbf{L}}_{G_{1}}\right)=\exp _{\mathrm{D}}^{-1}\left(\alpha \hat{\mathbf{L}}_{G_{2}}\right) \hat{\mathbf{L}}_{G_{1}-G_{2}} \exp _{\mathrm{D}}\left(\alpha \hat{\mathbf{L}}_{G_{1}}\right)= \\
& =\exp _{\mathrm{D}}^{-1}\left(\alpha \hat{\mathbf{L}}_{G_{2}}\right) \hat{\mathbf{L}}_{\hat{\mathbf{P}}_{H} F_{21}} \exp _{\mathrm{D}}\left(\alpha \hat{\mathbf{L}}_{G_{1}}\right)=\hat{\mathbf{L}}_{\exp _{\mathrm{D}}^{-1}\left(\alpha \hat{\mathbf{L}}_{G_{2}}\right) \hat{\mathbf{P}}_{H} F_{21}} \widehat{\mathbf{U}}_{21} .
\end{aligned}
$$

Согласно (31) разность между генераторами всегда имеет вид $\hat{\mathbf{P}}_{H} F_{21}$ с некоторой функцией $F_{21}(\mathbf{x}, \alpha)$. Благодаря соотношению (26) генератор $\widehat{\mathbf{U}}_{21}$ оказывается секулярным:

$$
G_{21}=\exp _{\mathrm{D}}^{-1}\left(\alpha \hat{\mathbf{L}}_{G_{2}}\right) \hat{\mathbf{P}}_{H} F_{21}=\hat{\mathbf{P}}_{H_{0}} \exp _{\mathrm{D}}^{-1}\left(\alpha \hat{\mathbf{L}}_{G_{2}}\right) F_{21}
$$

Мы можем заключить, что нормализованные в различных стилях гамильтонианы связаны преобразованием Ли-Депри с генератором, принадлежащим алгебре интегралов невозмущенной системы. Это соответствует теореме Брюно для производящих функций [32].

Для нерезонансных систем с несоизмеримыми частотами все интегралы коммутативны. Следовательно, нерезонансный нормализованный гамильтониан уникален и нечувствителен к процедуре нормализации [23].

Это не так в случае резонансов. Так как резонансные соотношения приводят к появлению некоммутативных интегралов, резонансный нормализованный гамильтониан зависит от стиля. Можно получить выражение для $\widetilde{H}$, следуя идее Виттота [46]. Рассмотрим производную

$$
\begin{aligned}
\frac{\partial}{\partial \alpha} \widetilde{H} & =\left(\frac{\partial}{\partial \alpha} \exp _{\mathrm{D}}^{-1}\left(\alpha \hat{\mathbf{L}}_{G}\right)\right) H+\exp _{\mathrm{D}}^{-1}\left(\alpha \hat{\mathbf{L}}_{G}\right) \frac{\partial}{\partial \alpha} H=\exp _{\mathrm{D}}^{-1}\left(\alpha \hat{\mathbf{L}}_{G}\right)\left(\hat{\mathbf{L}}_{H} G+H_{j}\right)= \\
& =\exp _{\mathrm{D}}^{-1}\left(\alpha \hat{\mathbf{L}}_{-\hat{\mathbf{S}}_{H} H_{j}+\hat{\mathbf{P}}_{H} F}\right) \hat{\mathbf{P}}_{H}\left(H_{j}+\hat{\mathbf{D}}_{H} F\right)= \\
& =\hat{\mathbf{P}}_{H_{0}} \exp _{\mathrm{D}}^{-1}\left(\alpha \hat{\mathbf{L}}_{-\hat{\mathbf{S}} H_{j}+\hat{\mathbf{P}}_{H} F}\right)\left(H_{j}+\hat{\mathbf{D}}_{H} F\right) .
\end{aligned}
$$

Здесь мы использовали уравнение (2) и каноническую связь проекторов (26). Следовательно,

$$
\widetilde{H}=H_{0}+\hat{\mathbf{P}}_{H_{0}} \int_{0}^{\alpha} \exp _{\mathrm{D}}^{-1}\left(\epsilon \hat{\mathbf{L}}_{-\hat{\mathbf{S}}_{H} H_{j}+\hat{\mathbf{P}}_{H} F}\right)\left(H_{j}+\hat{\mathbf{D}}_{H} F\right) d \epsilon .
$$

Это секулярное выражение демонстрирует явную зависимость от $F$.

5.3. Интегралы Густавсона. Интересно найти физически значимые величины, которые не будут зависеть от искусственного выбора стиля $F$. Рассмотрим систему с постоянными невозмущенными частотами. В своей знаменитой статье [10] Густавсон сконструировал формальные интегралы возмущенной системы, возникающие из центра алгебры интегралов невозмущенной системы. 
Каждое резонансное соотношение для невозмущенных частот $\left(\vec{\omega}, \vec{D}_{k}\right)=0$, где $k=1, \ldots, \mathbf{r}$, приводит к дополнительному некоммутативному интегралу. В представлениях Биркгофа и “действие-угол" центр соответствующей алгебры интегралов состоит из $\mathbf{d}-\mathbf{r}$ величин

$$
\tilde{I}_{m}=\sum_{l=1}^{\mathbf{d}} \beta_{m l} \widetilde{\zeta}_{l} \widetilde{\eta}_{l}=\left(\vec{\beta}_{m}, \vec{J}\right), \quad m=1, \mathbf{d}-\mathbf{r}
$$

Здесь $\vec{\beta}_{m}$ есть набор $\mathbf{d}-\mathbf{r}$ независимых векторов, ортогональных всем $\mathbf{r}$ резонансным векторам $\vec{D}_{k}$.

Эти интегралы коммутативны со всеми интегралами невозмущенной системы и, следовательно, с нормализованным гамильтонианом $\widetilde{H}$. В операторных обозначениях для любой аналитической функции $\widetilde{F}$

$$
\begin{gathered}
\tilde{I}_{m}=\hat{\mathbf{P}}_{H_{0}} \tilde{I}_{m}, \quad m=1, \mathbf{d}-\mathbf{r}, \\
{\left[\tilde{I}_{m}, \hat{\mathbf{P}}_{H_{0}} \tilde{F}\right]=0, \quad\left[\tilde{I}_{m}, \tilde{H}\right]=0 .}
\end{gathered}
$$

После преобразования обратно к начальным координатам величины

$$
I_{m}=\exp _{\mathrm{D}}\left(\alpha \hat{\mathbf{L}}_{G}\right) \tilde{I}_{m}, \quad m=1, \mathbf{d}-\mathbf{r},
$$

станут формальными интегралами возмущенной системы. Более того, эти интегралы Густавсона будут коммутативны со всеми функциями, принадлежащими образy $\hat{\mathbf{P}}_{H}$ :

$$
\begin{aligned}
& I_{m}=\exp _{\mathrm{D}}\left(\alpha \hat{\mathbf{L}}_{G}\right) \hat{\mathbf{P}}_{H_{0}} \tilde{I}_{m}=\hat{\mathbf{P}}_{H} \exp _{\mathrm{D}}\left(\alpha \hat{\mathbf{L}}_{G}\right) \tilde{I}_{m}=\hat{\mathbf{P}}_{H} I_{m}, \quad m=1, \mathbf{d}-\mathbf{r}, \\
& {\left[I_{m}, \hat{\mathbf{P}}_{H} F\right]=\exp _{\mathrm{D}}\left(\alpha \hat{\mathbf{L}}_{G}\right)\left[\tilde{I}_{m}, \hat{\mathbf{P}}_{H_{0}} \exp _{\mathrm{D}}^{-1}\left(\alpha \hat{\mathbf{L}}_{G}\right) F\right]=0} \\
& {\left[I_{m}, H\right]=\exp _{\mathrm{D}}\left(\alpha \hat{\mathbf{L}}_{G}\right)\left[\tilde{I}_{m}, \tilde{H}\right]=0 .}
\end{aligned}
$$

Здесь мы снова использовали каноническую связь проекторов (26). Благодаря этим свойствам производная $I_{m}(\alpha)$ не будет зависеть от стиля:

$$
\left(\frac{\partial}{\partial \alpha} I_{m}(\alpha)\right)=\left(\frac{\partial}{\partial \alpha} \exp _{\mathrm{D}}\left(\alpha \hat{\mathbf{L}}_{G}\right)\right) \tilde{I}_{m}=\hat{\mathbf{L}}_{G} I_{m}=-\hat{\mathbf{L}}_{\hat{\mathbf{s}}_{H} H_{j}} I_{m}+\hat{\mathbf{L}}_{\hat{\mathbf{p}}_{H} F} I_{m}=-\hat{\mathbf{L}}_{\hat{\mathbf{s}}_{H} H_{j}} I_{m} .
$$

Следовательно, интегралы Густавсона не чувствительны к стилю нормализации. Оказывается, ряды для этих величин расходятся [47], но тем не менее являются полезными для исследования областей регулярной динамики.

Невозмущенный гамильтониан $H_{0}$ может быть выбран в качестве начальной функции для $\tilde{I}_{m}$. Первые порядки нетривиальной части соответствующего интеграла Густавсона имеют вид

$$
\begin{aligned}
I_{G}= & \alpha^{-1}\left(H-\exp _{\mathrm{D}}\left(\alpha \hat{\mathbf{L}}-\hat{\mathbf{S}}_{H} H_{j}\right) H_{0}\right)=\hat{\mathbf{P}} H_{j}-\alpha\left(\hat{\mathbf{S}} \hat{\mathbf{L}} \hat{\mathbf{P}} H_{j}+\frac{1}{2} \hat{\mathbf{P}} \hat{\mathbf{L}} \hat{\mathbf{S}} H_{j}\right)+ \\
& +\alpha^{2}\left(\hat{\mathbf{S}} \hat{\mathbf{L}} \hat{\mathbf{S}} \hat{\mathbf{P}} H_{j}+\frac{1}{2} \hat{\mathbf{S}} \hat{\mathbf{L}} \hat{\mathbf{P}} \hat{\mathbf{S}} H_{j}+\frac{1}{3} \hat{\mathbf{P}} \hat{\mathbf{L}} \hat{\mathbf{L}} \hat{\mathbf{S}} H_{j}-\frac{2}{3} \hat{\mathbf{P}} \hat{\mathbf{L}} \hat{\mathbf{S}}^{2} \hat{\mathbf{L}} \hat{\mathbf{P}} H_{j}-\frac{1}{3} \hat{\mathbf{P}} \hat{\mathbf{L}} \hat{\mathbf{L}} \hat{\mathbf{S}}^{2} H_{j}\right)+ \\
& +O\left(\alpha^{3}\right) .
\end{aligned}
$$


Этот ряд применим и к системам с непостоянными невозмущенными частотами. Он известен как первый формальный интеграл Хори (см. также демонстрацию "Gustavson_jntegral").

ПримеР 2. СИСТЕмА ХЕНОНА-ХЕЙЛЕСА. Это двумерная система с гамильтонианом

$$
H=\frac{1}{2}\left(p_{1}^{2}+q_{1}^{2}+p_{2}^{2}+q_{2}^{2}\right)+\alpha\left(q_{1}^{2} q_{2}-\frac{1}{3} q_{2}^{3}\right) .
$$

В комплексных переменных $\zeta, \eta(9)$ гамильтониан Хенона-Хейлеса принимает вид

$$
\begin{aligned}
H= & i \zeta_{2} \eta_{2}+i \zeta_{1} \eta_{1}+\alpha \frac{1}{2 \sqrt{2}}\left(\zeta_{2} \eta_{2}^{2}-\frac{1}{3} \zeta_{2}^{3}-\eta_{1}^{2} \zeta_{2}-2 \zeta_{1} \eta_{1} \eta_{2}+\zeta_{1}^{2} \zeta_{2}+\right. \\
& \left.+\frac{1}{3} i \eta_{2}^{3}-i \zeta_{2}^{2} \eta_{2}-i \eta_{1}^{2} \eta_{2}+2 i \zeta_{1} \eta_{1} \zeta_{2}+i \zeta_{1}^{2} \eta_{2}\right)
\end{aligned}
$$

В первых порядках нормализованный гамильтониан имеет вид

$$
\begin{aligned}
\widetilde{H}= & i\left(\zeta_{2} \eta_{2}+i \zeta_{1} \eta_{1}\right)+\alpha^{2}\left(\frac{5}{12} \zeta_{2}^{2} \eta_{2}^{2}+\frac{7}{12} \eta_{1}^{2} \zeta_{2}^{2}-\frac{1}{3} \zeta_{1} \eta_{1} \zeta_{2} \eta_{2}+\frac{7}{12} \zeta_{1}^{2} \eta_{2}^{2}+\frac{5}{12} \zeta_{1}^{2} \eta_{1}^{2}\right)+ \\
& +i \alpha^{4}\left(\frac{235}{432} \zeta_{2}^{3} \eta_{2}^{3}-\frac{175}{144} \eta_{1}^{2} \zeta_{2}^{3} \eta_{2}-\frac{47}{16} \zeta_{1} \eta_{1} \zeta_{2}^{2} \eta_{2}^{2}+\frac{161}{144} \zeta_{1} \eta_{1}^{3} \zeta_{2}^{2}-\right. \\
& \left.-\frac{175}{144} \zeta_{1}^{2} \zeta_{2} \eta_{2}^{3}+\frac{65}{16} \zeta_{1}^{2} \eta_{1}^{2} \zeta_{2} \eta_{2}+\frac{161}{144} \zeta_{1}^{3} \eta_{1} \eta_{2}^{2}-\frac{101}{432} \zeta_{1}^{3} \eta_{1}^{3}\right)+O\left(\alpha^{6}\right)
\end{aligned}
$$

Из-за наличия резонанса $1: 1$ мы видим здесь смешанные члены, такие как $\zeta_{1} \eta_{2}$. Первые порядки интеграла Густавсона:

$$
\begin{aligned}
I_{G}= & \alpha^{-2}\left(H-\exp _{\mathrm{D}}\left(\alpha \hat{\mathbf{L}}_{G}\right) H_{0}\right)= \\
= & -\frac{1}{48}\left(5 p_{1}^{4}+2 p_{1}^{2}\left(5 p_{2}^{2}+5 q_{1}^{2}-9 q_{2}^{2}\right)+56 p_{1} p_{2} q_{1} q_{2}+5 p_{2}^{4}-2 p_{2}^{2}\left(9 q_{1}^{2}-5 q_{2}^{2}\right)+5\left(q_{1}^{2}+q_{2}^{2}\right)^{2}\right)- \\
& -\frac{\alpha}{36}\left(-28 p_{1}^{4} q_{2}+28 p_{1}^{3} p_{2} q_{1}+p_{1}^{2} q_{2}\left(84 p_{2}^{2}-27 q_{1}^{2}+37 q_{2}^{2}\right)+42 p_{1} p_{2} q_{1}\left(-2 p_{2}^{2}+q_{1}^{2}+q_{2}^{2}\right)-\right. \\
& \left.-p_{2}^{2}\left(69 q_{1}^{2} q_{2}+5 q_{2}^{3}\right)-5 q_{2}\left(q_{2}^{2}-3 q_{1}^{2}\right)\left(q_{1}^{2}+q_{2}^{2}\right)\right)+O\left(\alpha^{2}\right) .
\end{aligned}
$$

В этих порядках выражения идентичны соответствующим выражениям из классических работ [10], [48]. Дальнейшие порядки приведены в демонстрации "Henon_Heiles". Различия между нашими выражениями и классическими рядами Депри начинаются в пятом порядке генераторов и восьмом порядке нормализованных гамильтонианов.

Как и ожидалось, разность генераторов принадлежит ядру $1-\hat{\mathbf{P}}_{H}$, а нормализованные гамильтонианы связаны преобразованием Ли-Депри с секулярным генератором. Интегралы Густавсона идентичны вплоть до наивысшего рассчитывавшегося нами порядка.

Необходимо сказать несколько слов о нерезонансных системах. Теория КАМ [21], [49] показала, что нерезонансные динамические системы стабильны относительно небольших возмущений в том смысле, что большинство их инвариантных торов 
не разрушаются. В работах [50], [51] были продемонстрированы сходимость рядов Пуанкаре-Линдштедта на нерезонансном множестве и систематические сокращения членов с малыми знаменателями. Поэтому естественно искать нерезонансные сокращения и в рядах Като. Такие сокращения, связь с адельфическими интегралами Уиттекера и соответствующие вычислительные алгоритмы будут рассмотрены в отдельной работе.

\section{6. ВЫЧИСЛИТЕЛЬНЫЕ АСПЕКТЫ}

Главное различие между данным и классическими пертурбативными алгоритмами Депри [4], Хори [24], Драгта-Финна [5] и т. д. состоит в явных нерекуррентных формулах. Традиционно вычисления теории возмущений решают гомологические уравнения порядок за порядком. В отличие от них, мы напрямую вычисляем генератор $G=-\hat{\mathbf{S}}_{H} H_{j}$ до требуемого порядка в виде суммы перестановок (30). Это напоминает диаграммный подход в квантовой теории поля. Затем экспонента Депри нормализует гамильтониан. Детали явного "квадратного" алгоритма для генератора представлены нами в приложении В.

Явные выражения важны с общематематической точки зрения, так как они систематизируют и упрощают пертурбативные разложения за счет дополнительных секулярных членов в генераторе. Из-за этих членов наш подход будет менее эффективным для практических вычислений, чем классический алгоритм Депри. Тем не менее это не должно быть серьезной проблемой для современных компьютеров.

Мы сравнили компьютерное время, затраченное на прямую нормализацию с помощью явного выражения (30), с временнь́ми затратами для алгоритмов Депри [4] и Драгта-Финна [5]. Подход Драгта-Финна использует произведение канонических отображений

$$
\widetilde{H}=e^{-\alpha^{n} \hat{\mathbf{L}}_{G_{n-1}}} \cdots e^{-\alpha \hat{\mathbf{L}}_{G_{0}}} H, \quad \widetilde{\mathbf{x}}=e^{\alpha \hat{\mathbf{L}}_{G_{0}} \cdots e^{\alpha^{n} \hat{\mathbf{L}}_{G_{n-1}} \mathbf{X}}}
$$

вместо преобразований Ли-Депри для нормализации гамильтониана.

В табл. 1 приведено сравнение времен вычислений нормализованного гамильтониана для одномерного ангармонического осциллятора и двумерной системы ХенонаХейлеса на процессоре Intel Xeon X5675 (3.06 ГГц). Таблица демонстрирует, что наш метод действительно медленнее метода Депри, но остается достаточно эффективным для практических вычислений. Метод Драгта-Финна быстрее для одномерных систем и небольших порядков, но менее эффективен для расчета высоких порядков. Это следствие присущего ему возрастающего количества вычислений экспонент. Хотя число вычислений скобок Пуассона в методе Драгта-Финна меньше, чем в алгоритме Депри [1], операнды этих скобок содержат больше членов.

Все методы строят близкие к тождественному канонические нормализующие преобразования. Такие преобразования всегда являются гамильтоновыми потоками, эквивалентными преобразованиям Ли-Депри с различающимися стилями нормализации $\hat{\mathbf{P}}_{H} F$. Как и ожидалось, все три нормализованных гамильтониана отличаются друг от друга начиная с восьмого порядка. В то же время ряды для интегралов Густавсона совпадают. Преимуществом явной формулы (30) является возможность вычисления интегралов Густавсона без предварительной нормализации. 
ТАБлицА 1. Время нормализации (с).

\begin{tabular}{|c|c|c|c|c|c|c|}
\hline \multirow[b]{2}{*}{ Порядок } & \multicolumn{3}{|c|}{ Ангармонический осциллятор (1d) } & \multicolumn{3}{|c|}{ Система Хенона-Хейлеса (2d) } \\
\hline & $\begin{array}{c}\text { Подход } \\
\text { Депри }\end{array}$ & $\begin{array}{c}\text { Подход } \\
\text { Драгта- } \\
\text { Финна }\end{array}$ & $\begin{array}{c}\text { Явное } \\
\text { выражение }\end{array}$ & $\begin{array}{c}\text { Подход } \\
\text { Депри }\end{array}$ & $\begin{array}{c}\text { Подход } \\
\text { Драгта- } \\
\text { Финна }\end{array}$ & $\begin{array}{c}\text { Явное } \\
\text { выражение }\end{array}$ \\
\hline 4 & 0.02 & 0.01 & 0.04 & 0.05 & 0.04 & 0.1 \\
\hline 8 & 0.12 & 0.06 & 0.3 & 0.5 & 1.2 & 1.3 \\
\hline 16 & 1.7 & 0.8 & 6 & 19 & 139 & 42 \\
\hline 24 & 10.7 & 3.5 & 49 & 345 & 2231 & 558 \\
\hline 32 & 46.1 & 10.2 & 235 & 3155 & 20930 & 4332 \\
\hline
\end{tabular}

Отметим, что все расчеты были однопоточными. В случае необходимости сумма всех размещений в выражении (30) может быть параллелизована и сделана масштабируемой для современных многопроцессорных и облачных вычислений.

\section{7. НЕПЕРТУРБАТИВНЫЕ ПРИМЕРЫ}

Так как теперь у нас есть явное выражение для генератора преобразования Пуанкаре-Линдштедта, интересно применить его для точного решения и сконструировать явное выражение для $\hat{\mathrm{S}}_{H} H_{j}$. Это возможно только для тривиальных систем, поскольку требует алгебраической интегрируемости для всех $\alpha$. Тем не менее следующие игрушечные примеры хорошо иллюстрируют пертурбативные разложения.

ПРИМЕР 3. СДВИГ ЧАСТОТЫ ГАРМОНИЧЕСКОГО ОСЦИЛЛЯТОРА. ГамиЛЬтониан имеет вид

$$
H=\frac{1}{2}\left(p^{2}+q^{2}\right)+\frac{\alpha}{2} q^{2} .
$$

Здесь мы знаем точное решение

$$
q(t)=\sqrt{\frac{2 E}{1+\alpha}} \cos (\sqrt{1+\alpha} t+\phi),
$$

где постоянные энергии $E=H(p, q)$ и фазы $\phi$ суть функции начальной точки $\left(p_{0}, q_{0}\right)$.

Мы можем получить “точные" пертурбативные операторы при помощи выражений (17), а затем подставить $p_{0} \rightarrow p, q_{0} \rightarrow q$ :

$$
\begin{aligned}
& \hat{\mathbf{P}}_{H} \frac{q^{2}}{2}=\lim _{\lambda \rightarrow+0} \lambda \int_{0}^{+\infty} e^{-\lambda t} \frac{q(t)^{2}}{2} d t=\frac{H}{2(1+\alpha)}, \\
& \hat{\mathbf{S}}_{H} \frac{q^{2}}{2}=-\lim _{\lambda \rightarrow+0} \int_{0}^{\infty} e^{-\lambda t}\left(\frac{q(t)^{2}}{2}-\hat{\mathbf{P}}_{H} H_{j}\right) d t=-\frac{p q}{4(1+\alpha)} .
\end{aligned}
$$

Действительно,

$$
\hat{\mathbf{L}}_{H}\left(-\frac{p q}{4(1+\alpha)}\right)=\frac{-p^{2}+q^{2}(1+\alpha)}{4(1+\alpha)}=\frac{q^{2}}{2}-\hat{\mathbf{P}}_{H} \frac{q^{2}}{2} .
$$


Следовательно, точный генератор $G=p q / 4(1+\alpha)$. Нормализующее каноническое преобразование (экспонента Депри) определяется уравнениями

$$
\begin{aligned}
& \frac{\partial p}{\partial \alpha}=\hat{\mathbf{L}}_{G} p=-\frac{p}{4(1+\alpha)} \\
& \frac{\partial q}{\partial \alpha}=\hat{\mathbf{L}}_{G} q=\frac{q}{4(1+\alpha)}
\end{aligned}
$$

имеющими решение

$$
\tilde{p}=\frac{1}{\sqrt[4]{1+\alpha}} p, \quad \tilde{q}=\sqrt[4]{1+\alpha} q
$$

Нормализованный гамильтониан $\widetilde{H}=(\sqrt{1+\alpha} / 2)\left(\tilde{p}^{2}+\tilde{q}^{2}\right)$. Степенное разложение этой формулы совпадает со стандартным рядом теории возмущений.

ПримеР 4. УРАВНЕНИЕ ДЮФФИНГА (продолжение примера 1). Гамильтониан имеет вид

$$
H=\frac{1}{2}\left(p^{2}+q^{2}\right)+\frac{\alpha}{4} q^{4}
$$

Эта система имеет точное решение в эллиптических функциях Якоби:

$$
\begin{gathered}
q(t)=A \operatorname{cn}\left(\omega t+\psi, k^{2}\right), \\
\omega^{2}=\sqrt{1+4 \alpha E}, \quad A^{2}=\frac{1}{\alpha}\left(\omega^{2}-1\right), \quad k^{2}=\frac{1}{2}\left(1-\frac{1}{\omega^{2}}\right) .
\end{gathered}
$$

Здесь энергия $E=H\left(p_{0}, q_{0}\right)$, а псевдофаза $\psi$ определяется начальным условием

$$
\frac{\operatorname{sn}\left(\psi, k^{2}\right)}{\operatorname{cn}\left(\psi, k^{2}\right)} \operatorname{dn}\left(\psi, k^{2}\right)=-\frac{p_{0}}{q_{0} \omega}
$$

Чтобы найти точный интегрирующий оператор $\hat{\mathbf{S}}_{H} H_{j}$, мы можем разложить возмущение $H_{j}=q^{4} / 4$ в ряд Фурье при помощи выражения [52]

$$
\mathrm{cn}^{4}\left(u, k^{2}\right)=\mathrm{const}+\frac{4 \pi^{2}}{3 k^{4} \mathrm{~K}^{2}} \sum_{n=1}^{\infty} \frac{n Q^{n}}{1-Q^{2 n}}\left(\left(2 k^{2}-1\right)+\frac{n^{2} \pi^{2}}{4 \mathrm{~K}^{2}}\right) \cos \left(\frac{n \pi}{\mathrm{K}} u\right) .
$$

Здесь $\mathrm{K}\left(k^{2}\right)$ - полный эллиптический интеграл первого рода, a

$$
Q=\exp \left(-\frac{\pi \mathrm{K}\left(k^{2}\right)}{\mathrm{K}\left(1-k^{2}\right)}\right)
$$

есть эллиптический “ном”. 
"Точный" нормализующий генератор имеет вид

$$
\begin{aligned}
G & =-\hat{\mathbf{S}}_{H} H_{j}=\lim _{\lambda \rightarrow+0} \int_{0}^{\infty} e^{-\lambda t}\left(\frac{q(t)^{4}}{4}-\hat{\mathbf{P}}_{H} H_{j}\right) d t= \\
& =-\frac{\pi A^{4}}{3 k^{4} \omega \mathrm{K}} \sum_{n=1}^{\infty} \frac{Q^{n}}{1-Q^{2 n}}\left(\frac{n^{2} \pi^{2}}{4 \mathrm{~K}^{2}}-\frac{1}{\omega^{2}}\right) \sin \left(\frac{n \pi}{\mathrm{K}} \psi\right)
\end{aligned}
$$

Для сравнения со стандартным разложением введем $2 \pi$-периодическую псевдофазу $\theta=\pi \psi / 2 \mathrm{~K}$. Этот угол может быть получен из (38) в виде степенного ряда (здесь мы снова подставили $p_{0} \rightarrow p, q_{0} \rightarrow q$ ):

$$
\operatorname{tg} \theta=-\frac{p}{q}+\frac{p\left(3 p^{2}+5 q^{2}\right)}{4 q\left(p^{2}+q^{2}\right)} k^{2}+O\left(k^{4}\right) .
$$

Теперь можно разложить “точную” формулу (39) в степенной ряд при помощи системы алгебраических вычислений Mathematica:

$$
G=\frac{p q}{32}\left(3 p^{2}+5 q^{2}\right)-\alpha \frac{p q}{384}\left(39 p^{4}+104 p^{2} q^{2}+57 q^{4}\right)+O\left(\alpha^{2}\right)
$$

Это выражение совпадает с рядом теории возмущений (34). Интересно, что разложение "точного" ряда Фурье потребовало значительно больше компьютерного времени, чем расчет по теории возмущений.

\section{8. ЗАКЛЮЧЕНИЕ}

В настоящей работе мы применили резольвентное разложение Като к классической механике. Канонические тождества для усредняющего и интегрирующего операторов выявили регулярную структуру в пертурбативных рядах. Для объяснения этой структуры использовалась связь пертурбативных операторов с коэффициентами ряда Лорана резольвенты оператора Лиувилля.

Ряды Като и резольвентное каноническое тождество систематизируют разложения и приводят к новому явному выражению для генератора Депри преобразования Пуанкаре-Линдштедта в любом порядке: $G=-\hat{\mathbf{S}}_{H} H_{j}$. Здесь интегрирующий оператор $\hat{\mathbf{S}}_{H}$ является частичным псевдообратным к возмущенному оператору Лиувилля. Мы использовали непертурбативные примеры для иллюстрации этой формулы.

После распространения формализма на многомерные системы мы обсудили неоднозначности генератора и нормализованного гамильтониана. Интересно, что интегралы Густавсона оказались нечувствительными к процедуре нормализации.

Все обсуждение проводилось на формальном уровне. Сравнение вычислительных затрат данного подхода и классических алгоритмов Депри и Драгта-Финна продемонстрировали достаточную эффективность алгоритма даже для высоких порядков теории возмущений. 


\section{ПРИЛОЖКНИЕ А}

\section{Свойства возмущенных операторов}

Исследуем свойства усеченных пертурбативных операторов $\hat{\mathbf{P}}_{H}^{[N]}, \hat{\mathbf{S}}_{H}^{[N]}$ и $\hat{\mathbf{D}}_{H}^{[N]}$ :

$$
\begin{aligned}
\hat{\mathbf{P}}_{H}^{[N]} & =\sum_{n=0}^{N}(-1)^{n+1} \alpha^{n}(\sum_{\substack{\sum_{p_{j}=\mathbf{n}} \\
p_{j} \geqslant 0}} \hat{\mathbf{R}}_{H_{0}}^{\left(p_{n+1}\right)} \underbrace{\hat{\mathbf{L}}_{H_{j}} \hat{\mathbf{R}}_{H_{0}}^{\left(p_{n}\right)} \ldots \hat{\mathbf{R}}_{H_{0}}^{\left(p_{2}\right)} \hat{\mathbf{L}}_{H_{j}}}_{n \text { раз }} \hat{\mathbf{R}}_{H_{0}}^{\left(p_{1}\right)}), \\
\hat{\mathbf{S}}_{H}^{[N]} & =\sum_{n=0}^{N}(-1)^{n} \alpha^{n}(\sum_{\substack{\sum p_{j}=\mathbf{n}+\mathbf{1} \\
p_{j} \geqslant 0}} \hat{\mathbf{R}}_{H_{0}}^{\left(p_{n+1}\right)} \underbrace{\hat{\mathbf{L}}_{H_{j}} \hat{\mathbf{R}}_{H_{0}}^{\left(p_{n}\right)} \ldots \hat{\mathbf{R}}_{H_{0}}^{\left(p_{2}\right)} \hat{\mathbf{L}}_{H_{j}}}_{n \text { раз }} \hat{\mathbf{R}}_{H_{0}}^{\left(p_{1}\right)}), \\
\hat{\mathbf{D}}_{H}^{[N]} & =\sum_{n=1}^{N}(-1)^{n+1} \alpha^{n}(\sum_{\substack{\sum p_{j}=\mathbf{n}-\mathbf{1} \\
p_{j} \geqslant 0}} \hat{\mathbf{R}}_{H_{0}}^{\left(p_{n+1}\right)} \underbrace{\hat{\mathbf{L}}_{H_{j}} \hat{\mathbf{R}}_{H_{0}}^{\left(p_{n}\right)} \ldots \hat{\mathbf{R}}_{H_{0}}^{\left(p_{2}\right)} \hat{\mathbf{L}}_{H_{j}}}_{n \text { раз }} \hat{\mathbf{R}}_{H_{0}}^{\left(p_{1}\right)})
\end{aligned}
$$

(здесь обозначение $[N]$ - индекс). Мы будем подразумевать $N \geqslant 2$. Полезно ввести операторы

$$
\hat{\mathbf{Z}}_{n}^{m}= \begin{cases}(-1)^{n+1} \sum_{\substack{p_{1}+\ldots+p_{n+1}=m, p_{j} \geqslant 0}} \hat{\mathbf{R}}_{H_{0}}^{\left(p_{n+1}\right)} \underbrace{\hat{\mathbf{L}}_{H_{j}} \hat{\mathbf{R}}_{H_{0}}^{\left(p_{n}\right)} \ldots \hat{\mathbf{R}}_{H_{0}}^{\left(p_{2}\right)} \hat{\mathbf{L}}_{H_{j}}}_{n \text { раз }} \hat{\mathbf{R}}_{H_{0}}^{\left(p_{1}\right)}, & m \geqslant 0, \\ 0, & m<0 .\end{cases}
$$

Суммирование в них проходит по всем размещениям $m$ операторов $\hat{\mathbf{S}}_{H_{0}}$ в $n+1$ мест.

Важно, что данные операторы могут быть вычислены рекуррентно. Для любого $k, 0 \leqslant k \leqslant n-1$,

$$
\hat{\mathbf{Z}}_{n}^{m}=\sum_{l=0}^{m} \hat{\mathbf{Z}}_{n-k-1}^{m-l} \hat{\mathbf{L}}_{H_{j}} \hat{\mathbf{Z}}_{k}^{l} .
$$

Простейшими такими операторами являются $\hat{\mathbf{Z}}_{0}^{0}=\hat{\mathbf{P}}$ и $\hat{\mathbf{Z}}_{0}^{m}=-\hat{\mathbf{S}}^{m}$ для $m>0$. Следовательно,

$$
\begin{aligned}
& \hat{\mathbf{Z}}_{0}^{m} \hat{\mathbf{L}}_{H_{0}}=\hat{\mathbf{L}}_{H_{0}} \hat{\mathbf{Z}}_{0}^{m}=\hat{\mathbf{Z}}_{0}^{m-1}-\delta_{1}^{m}, \\
& \hat{\mathbf{Z}}_{0}^{m} H_{0}=H_{0} \delta_{0}^{m},
\end{aligned}
$$

где $\delta_{1}^{m}-$ символ Кронекера. Для $n \geqslant 1$ имеем

$$
\begin{aligned}
& \hat{\mathbf{Z}}_{n}^{m} \hat{\mathbf{L}}_{H_{0}}=\sum_{l=0}^{m} \hat{\mathbf{Z}}_{n-1}^{m-l} \hat{\mathbf{L}}_{H_{j}} \hat{\mathbf{Z}}_{0}^{l} \hat{\mathbf{L}}_{H_{0}}=\sum_{l=1}^{m} \hat{\mathbf{Z}}_{n-1}^{m-l} \hat{\mathbf{L}}_{H_{j}} \hat{\mathbf{Z}}_{0}^{l-1}-\hat{\mathbf{Z}}_{n-1}^{m-1} \hat{\mathbf{L}}_{H_{j}}=\hat{\mathbf{Z}}_{n}^{m-1}-\hat{\mathbf{Z}}_{n-1}^{m-1} \hat{\mathbf{L}}_{H_{j}}, \\
& \hat{\mathbf{L}}_{H_{0}} \hat{\mathbf{Z}}_{n}^{m}=\sum_{l=0}^{m} \hat{\mathbf{L}}_{H_{0}} \hat{\mathbf{Z}}_{0}^{l} \hat{\mathbf{L}}_{H_{j}} \hat{\mathbf{Z}}_{n-1}^{m-l}=\sum_{l=1}^{m} \hat{\mathbf{Z}}_{0}^{l-1} \hat{\mathbf{L}}_{H_{j}} \hat{\mathbf{Z}}_{n-1}^{m-l}-\hat{\mathbf{L}}_{H_{j}} \hat{\mathbf{Z}}_{n-1}^{m-1}=\hat{\mathbf{Z}}_{n}^{m-1}-\hat{\mathbf{L}}_{H_{j}} \hat{\mathbf{Z}}_{n-1}^{m-1}, \\
& \hat{\mathbf{Z}}_{n}^{m} H_{0}=\sum_{l=0}^{m} \hat{\mathbf{Z}}_{n-1}^{m-l} \hat{\mathbf{L}}_{H_{j}} \hat{\mathbf{Z}}_{0}^{l} H_{0}=\hat{\mathbf{Z}}_{n-1}^{m} \hat{\mathbf{L}}_{H_{j}} H_{0}=-\hat{\mathbf{Z}}_{n-1}^{m} \hat{\mathbf{L}}_{H_{0}} H_{j}=-\hat{\mathbf{Z}}_{n-1}^{m-1} H_{j}+\delta_{1}^{n} \delta_{1}^{m} H_{j} .
\end{aligned}
$$


Рассмотрим конечные операторные суммы с двумя индексами:

$$
\hat{\mathbf{R}}_{H}^{[N](k)}=-\sum_{n=0}^{N} \alpha^{n} \hat{\mathbf{Z}}_{n}^{n+k}
$$

Они имеют следующее асимптотическое поведение:

$$
\hat{\mathbf{R}}_{H}^{[N](k)}= \begin{cases}\hat{\mathbf{S}}^{k}+O(\alpha), & k \geqslant 1 \\ -\hat{\mathbf{P}}+O(\alpha), & k=0 \\ O\left(\alpha^{k}\right), & k<0\end{cases}
$$

Эти суммы унифицируют выражения для операторов

$$
\hat{\mathbf{P}}_{H}^{[N]}=-\hat{\mathbf{R}}_{H}^{[N](0)}, \quad \hat{\mathbf{S}}_{H}^{[N]}=\hat{\mathbf{R}}_{H}^{[N](1)}, \quad \hat{\mathbf{D}}_{H}^{[N]}=-\hat{\mathbf{R}}_{H}^{[N](-1)} .
$$

Их действия на возмущенный гамильтониан $H=H_{0}+\alpha H_{j}$ следующие:

$$
\begin{aligned}
\hat{\mathbf{R}}_{H}^{[N](k)} \hat{\mathbf{L}}_{H} & =-\hat{\mathbf{Z}}_{0}^{k} \hat{\mathbf{L}}_{H_{0}}-\sum_{n=1}^{N} \alpha^{n}\left(\hat{\mathbf{Z}}_{n}^{n+k} \hat{\mathbf{L}}_{H_{0}}+\hat{\mathbf{Z}}_{n-1}^{n+k-1} \hat{\mathbf{L}}_{H_{j}}\right)+O\left(\alpha^{N+1}\right)= \\
& =\delta_{1}^{k}+\hat{\mathbf{R}}_{H}^{[N](k-1)}+O\left(\alpha^{N+1}\right), \\
\hat{\mathbf{L}}_{H} \hat{\mathbf{R}}_{H}^{[N](k)} & =\ldots=\delta_{1}^{k}+\hat{\mathbf{R}}_{H}^{[N](k-1)}+O\left(\alpha^{N+1}\right), \\
\hat{\mathbf{R}}_{H}^{[N](k)} H & =-\hat{\mathbf{Z}}_{0}^{k} H_{0}-\sum_{n=1}^{N} \alpha^{n}\left(\hat{\mathbf{Z}}_{n}^{n+k} H_{0}+\hat{\mathbf{Z}}_{n-1}^{n+k-1} H_{j}\right)+O\left(\alpha^{N+1}\right)= \\
& =-\delta_{0}^{k} H+O\left(\alpha^{N+1}\right) .
\end{aligned}
$$

Рассмотрим теперь резольвентный ряд, усеченный в порядках $N$ по $\alpha$ и $M$ по $z$ :

$$
\hat{\boldsymbol{R}}_{H}^{[N, M]}(z)=\sum_{k=-N}^{M+1} \hat{\mathbf{R}}_{H}^{[N](k)} z^{k-1}=-\sum_{k=-N-1}^{M} z^{k}\left(\sum_{n=0}^{N} \alpha^{n} \hat{\mathbf{Z}}_{n}^{n+k+1}\right) .
$$

Здесь $\hat{\mathcal{R}}_{H}^{[N, M]}(z)$ является явно мероморфной функцией комплексного $z$. Мы будем использовать ее так же, как в теории вероятностей используют производящую функцию. Степени z будут служить для одновременного преобразования выражений. Благодаря тождествам (43) имеем

$$
\hat{\mathcal{R}}_{H}^{[N, M]}(z) \hat{\mathbf{L}}_{H}=\sum_{k=-N}^{M+1} z^{k-1} \hat{\mathbf{R}}_{H}^{[N](k)} \hat{\mathbf{L}}_{H}=1+z \hat{\boldsymbol{R}}_{H}^{[N, M]}(z)-z^{M+1} \hat{\mathbf{R}}_{H}^{[N](M+1)}+O\left(\alpha^{N+1}\right),
$$

$\hat{\mathbf{L}}_{H} \hat{\mathcal{R}}_{H}^{[N, M]}(z)=1+z \hat{\mathcal{R}}_{H}^{[N, M]}(z)-z^{M+1} \hat{\mathbf{R}}_{H}^{[N](M+1)}+O\left(\alpha^{N+1}\right)$.

Здесь член с $z^{M+1}$ возникает из-за краевого эффекта усечения. Поэтому мы можем 
аппроксимировать единичный оператор выражениями

$$
\begin{aligned}
& \hat{\boldsymbol{R}}_{H}^{[N, M]}(z)\left(\hat{\mathbf{L}}_{H}-z\right)+z^{M+1} \hat{\mathbf{R}}_{H}^{[N](M+1)}=1+O\left(\alpha^{N+1}\right), \\
& \left(\hat{\mathbf{L}}_{H}-z\right) \hat{\boldsymbol{\mathcal { R }}}_{H}^{[N, M]}(z)+z^{M+1} \hat{\mathbf{R}}_{H}^{[N](M+1)}=1+O\left(\alpha^{N+1}\right) .
\end{aligned}
$$

Соответствующее приближенное тождество Гильберта выглядит следующим образом:

$$
\begin{aligned}
\hat{\boldsymbol{R}}_{H}^{[N, M]} & \left(z_{1}\right)-\hat{\boldsymbol{\mathcal { R }}}_{H}^{[N, M]}\left(z_{2}\right)=\left(z_{1}-z_{2}\right) \hat{\boldsymbol{\mathcal { R }}}_{H}^{[N, M]}\left(z_{1}\right) \hat{\boldsymbol{\mathcal { R }}}_{H}^{[N, M]}\left(z_{2}\right)- \\
& -z_{1}^{M+1} \hat{\mathbf{R}}_{H}^{[N](M+1)} \hat{\boldsymbol{\mathcal { R }}}_{H}^{[N, M]}\left(z_{2}\right)+z_{2}^{M+1} \hat{\boldsymbol{\mathcal { R }}}_{H}^{[N, M]}\left(z_{1}\right) \hat{\mathbf{R}}_{H}^{[N](M+1)}+O\left(\alpha^{N+1}\right) .
\end{aligned}
$$

Можно получить выражение для произведения коэффициентов, соответствующее формуле (21),

$$
\begin{aligned}
\hat{\mathbf{R}}_{H}^{[N](m)} \hat{\mathbf{R}}_{H}^{[N](n)}= & \left(\frac{1}{2 \pi i}\right)^{2} \oint_{\left|z_{1}\right|=\epsilon_{1}} \oint_{\left|z_{2}\right|=\epsilon_{2}} \hat{\mathcal{R}}_{H}^{[N, M]}\left(z_{1}\right) \hat{\mathcal{R}}_{H}^{[N, M]}\left(z_{2}\right) z_{1}^{-m} z_{2}^{-n} d z_{1} d z_{2}= \\
= & \left(\eta_{m}+\eta_{n}-1\right) \hat{\mathbf{R}}_{H}^{[N](m+n)}-\eta_{m-M-1} \hat{\mathbf{R}}_{H}^{[N](M+1)} \hat{\mathbf{R}}_{H}^{[N](m+n-M-1)}+ \\
& +\left(1-\eta_{n-M-1}\right) \hat{\mathbf{R}}_{H}^{[N](m+n-M-1)} \hat{\mathbf{R}}_{H}^{[N](M+1)}+O\left(\alpha^{N+1}\right) .
\end{aligned}
$$

Для любых $m$ и $n$ мы можем выбрать порядок усечения $M$ таким, что $M \geqslant m+n+N$ и $\hat{\mathbf{R}}_{H}^{[N](m+n-M-1)}=O\left(\alpha^{N+1}\right)$. Следовательно,

$$
\hat{\mathbf{R}}_{H}^{[N](m)} \hat{\mathbf{R}}_{H}^{[N](n)}=\left(\eta_{m}+\eta_{n}-1\right) \hat{\mathbf{R}}_{H}^{[N](m+n)}+O\left(\alpha^{N+1}\right) .
$$

Из формул (43) и тождества (44) можно сделать следующие заключения.

1. Конечная сумма $\hat{\mathbf{P}}_{H}^{[N]}$ аппроксимирует проектор: $\hat{\mathbf{P}}_{H}^{[N]} \hat{\mathbf{P}}_{H}^{[N]}=\hat{\mathbf{P}}_{H}^{[N]}+O\left(\alpha^{N+1}\right)$.

2. Суммы $\hat{\mathbf{P}}_{H}^{[N]}, \hat{\mathbf{S}}_{H}^{[N]}$ и $\hat{\mathbf{D}}_{H}^{[N]}$ подчиняются приблизительно тем же тождествам, что и соответствующие невозмущенные операторы:

$$
\begin{array}{ll}
\hat{\mathbf{P}}_{H}^{[N]} \hat{\mathbf{S}}_{H}^{[N]}=0+O\left(\alpha^{N+1}\right), & \hat{\mathbf{S}}_{H}^{[N]} \hat{\mathbf{P}}_{H}^{[N]}=0+O\left(\alpha^{N+1}\right), \\
\hat{\mathbf{D}}_{H}^{[N]} \hat{\mathbf{S}}_{H}^{[N]}=0+O\left(\alpha^{N+1}\right), & \hat{\mathbf{S}}_{H}^{[N]} \hat{\mathbf{D}}_{H}^{[N]}=0+O\left(\alpha^{N+1}\right), \\
\hat{\mathbf{P}}_{H}^{[N]} \hat{\mathbf{D}}_{H}^{[N]}=\hat{\mathbf{D}}_{H}^{[N]}+O\left(\alpha^{N+1}\right), & \hat{\mathbf{D}}_{H}^{[N]} \hat{\mathbf{P}}_{H}^{[N]}=\hat{\mathbf{D}}_{H}^{[N]}+O\left(\alpha^{N+1}\right) .
\end{array}
$$

3. Эти операторы действуют на возмущенный гамильтониан $H=H_{0}+\alpha H_{j}$ следующим образом:

$$
\begin{gathered}
\hat{\mathbf{P}}_{H}^{[N]} H=H+O\left(\alpha^{N+1}\right), \quad \hat{\mathbf{S}}_{H}^{[N]} H=0+O\left(\alpha^{N+1}\right), \\
\hat{\mathbf{D}}_{H}^{[N]} H=0+O\left(\alpha^{N+1}\right) .
\end{gathered}
$$


4. Взаимодействия этих сумм с оператором Лиувилля следующие:

$$
\begin{array}{lll}
\hat{\mathbf{L}}_{H} \hat{\mathbf{P}}_{H}^{[N]}=\hat{\mathbf{D}}_{H}^{[N]}+O\left(\alpha^{N+1}\right), & \hat{\mathbf{P}}_{H}^{[N]} \hat{\mathbf{L}}_{H}=\hat{\mathbf{D}}_{H}^{[N]}+O\left(\alpha^{N+1}\right), \\
\hat{\mathbf{S}}_{H}^{[N]} \hat{\mathbf{L}}_{H}=1-\hat{\mathbf{P}}_{H}^{[N]}+O\left(\alpha^{N+1}\right), & \hat{\mathbf{L}}_{H} \hat{\mathbf{S}}_{H}^{[N]}=1-\hat{\mathbf{P}}_{H}^{[N]}+O\left(\alpha^{N+1}\right), \\
\hat{\mathbf{L}}_{H} \hat{\mathbf{D}}_{H}^{[N]}=\left(\hat{\mathbf{D}}_{H}^{[N]}\right)^{2}+O\left(\alpha^{N+1}\right), & \hat{\mathbf{D}}_{H}^{[N]} \hat{\mathbf{L}}_{H}=\left(\hat{\mathbf{D}}_{H}^{[N]}\right)^{2}+O\left(\alpha^{N+1}\right) .
\end{array}
$$

5. Степени операторов $\hat{\mathbf{S}}_{H}^{[N]}$ и $\hat{\mathbf{D}}_{H}^{[N]}$ могут быть записаны как

$$
\begin{gathered}
\left(\hat{\mathbf{S}}_{H}^{[N]}\right)^{k}=\hat{\mathbf{R}}_{H}^{[N](k)}+O\left(\alpha^{N+1}\right)=-\sum_{n=0}^{N} \alpha^{n} \hat{\mathbf{Z}}_{n}^{n+k}+O\left(\alpha^{N+1}\right), \\
\left(\hat{\mathbf{D}}_{H}^{[N]}\right)^{k}=-\hat{\mathbf{R}}_{H}^{[N](-k)}+O\left(\alpha^{N+1}\right)=\sum_{n=k}^{N} \alpha^{n} \hat{\mathbf{Z}}_{n}^{n-k}+O\left(\alpha^{N+1}\right) .
\end{gathered}
$$

Демонстрация "perturbed_operators" иллюстрирует приведенные выше тождества.

ПРИЛОЖЕНИЕ Б

\section{Каноническая связь проекторов}

Теперь мы используем усеченную резольвенту

$$
\begin{aligned}
\hat{\boldsymbol{R}}_{H}^{[N]}(z) & =-\frac{1}{z} \hat{\mathbf{P}}_{H}^{[N]}+\sum_{k=0}^{2 N} z^{k}\left(\hat{\mathbf{S}}_{H}^{[N]}\right)^{k+1}-\sum_{k=2}^{N+1} z^{-k}\left(\hat{\mathbf{D}}_{H}^{[N]}\right)^{k-1}= \\
& =-\sum_{k=-N-1}^{2 N} z^{k}\left(\sum_{n=0}^{N} \alpha^{n} \hat{\mathbf{Z}}_{n}^{n+k+1}\right),
\end{aligned}
$$

для того чтобы доказать каноническую связь между возмущенным и невозмущенным проекторами. В этом разделе мы установили порядок усечения по $z$ в $2 N$. Этого будет достаточно, чтобы избежать краевых эффектов, возникающих в выражении

$$
\hat{\mathcal{R}}_{H}^{[N]}(z)\left(\hat{\mathbf{L}}_{H}-z\right)+z^{2 N+1}\left(\hat{\mathbf{S}}_{H}^{[N]}\right)^{2 N+1}=1+O\left(\alpha^{N+1}\right) .
$$

В частности, так как $\left(\hat{\mathbf{D}}_{H}^{[N]}\right)^{m}=O\left(\alpha^{m}\right)$ и $\hat{\mathbf{S}}_{H}^{[N]} \hat{\mathbf{D}}_{H}^{[N]}=O\left(\alpha^{N+1}\right)$, то для всех целых $1 \leqslant k \leqslant N$ выполняется

$$
\operatorname{Res}_{z=0}\left(z^{2 N+1}\left(\hat{\mathcal{R}}_{H}^{[N]}(z)\right)^{k}\right)=O\left(\alpha^{N+1}\right)
$$

Кроме того, для любого целого $k \geqslant 2$

$$
\operatorname{Res}_{z=0}\left(\left(\hat{\mathcal{R}}_{H}^{[N]}(z)\right)^{k}\right)=O\left(\alpha^{N+1}\right)
$$


Как и ожидалось, производная проектора может быть получена как вычет:

$$
\begin{aligned}
& \operatorname{Res}_{z=0}\left(\hat{\boldsymbol{\mathcal { R }}}_{H}^{[N]}(z) \hat{\mathbf{L}}_{H_{j}} \hat{\boldsymbol{\mathcal { R }}}_{H}^{[N]}(z)\right)=\sum_{k=-N-1}^{N} \sum_{n=0}^{N} \sum_{m=0}^{N} \alpha^{n+m} \hat{\mathbf{Z}}_{n}^{n+k+1} \hat{\mathbf{L}}_{H_{j}} \hat{\mathbf{Z}}_{m}^{m-k}= \\
& =\sum_{n=0}^{N} \sum_{m=0}^{N} \alpha^{n+m} \hat{\mathbf{Z}}_{n+m+1}^{n+m+1}=\sum_{k=0}^{N}(k+1) \alpha^{k} \hat{\mathbf{Z}}_{k+1}^{k+1}+O\left(\alpha^{N+1}\right)=\left(\frac{\partial}{\partial \alpha} \hat{\mathbf{P}}_{H}\right)^{[N]}+O\left(\alpha^{N+1}\right) .
\end{aligned}
$$

Здесь не происходит потери точности, так как ряд усекается после дифференцирования.

Теперь мы можем построить конечный аналог канонического резольвентного тождества (27). Действие почти единичного оператора (45) на скобку Пуассона $\left[\hat{\mathcal{R}}_{H}^{[N]}\left(z_{2}\right) H_{j}, \hat{\mathcal{R}}_{H}^{[N]}(z) G\right]$ дает

$$
\begin{aligned}
& \hat{\mathcal{R}}_{H}^{[N]}(z) \hat{\mathbf{L}}_{H_{j}} \hat{\boldsymbol{\mathcal { R }}}_{H}^{[N]}(z)=\hat{\mathbf{L}}_{\hat{\mathcal{R}}_{H}^{[N]}\left(z_{2}\right) H_{j}} \hat{\boldsymbol{\mathcal { R }}}_{H}^{[N]}(z)-\hat{\mathcal{R}}_{H}^{[N]}(z) \hat{\mathbf{L}}_{\hat{\mathcal{R}}_{H}^{[N]}\left(z_{2}\right) H_{j}}- \\
& -z_{2} \hat{\boldsymbol{\mathcal { R }}}_{H}^{[N]}(z) \hat{\mathbf{L}}_{\hat{\mathcal{R}}_{H}^{[N]}\left(z_{2}\right) H_{j}} \hat{\boldsymbol{\mathcal { R }}}_{H}^{[N]}(z)-z^{2 N+1}\left(\left(\hat{\mathbf{S}}_{H}^{[N]}\right)^{2 N+1} \hat{\mathbf{L}}_{\hat{\mathcal{R}}_{H}^{[N]}\left(z_{2}\right) H_{j}} \hat{\mathcal{R}}_{H}^{[N]}(z)-\right. \\
& \left.-\hat{\mathcal{R}}_{H}^{[N]}(z) \hat{\mathbf{L}}_{\hat{\mathcal{R}}_{H}^{[N]}\left(z_{2}\right) H_{j}}\left(\hat{\mathbf{S}}_{H}^{[N]}\right)^{2 N+1}\right)+z_{2}^{2 N+1} \hat{\mathcal{R}}_{H}^{[N]}(z) \hat{\mathbf{L}}_{\left(\hat{\mathbf{S}}_{H}^{[N]}\right)^{2 N+1}{ }_{H_{j}} \hat{\mathcal{R}}_{H}^{[N]}(z)+} \\
& +O\left(\alpha^{N+1}\right) .
\end{aligned}
$$

Нам интересен вычет предыдущей формулы в $z=0$ :

$$
\begin{aligned}
& \left(\frac{\partial}{\partial \alpha} \hat{\mathbf{P}}_{H}\right)^{[N]}=\hat{\mathbf{P}}_{H}^{[N]} \hat{\mathbf{L}}_{\hat{\mathcal{R}}_{H}^{[N]}\left(z_{2}\right) H_{j}}-\hat{\mathbf{L}}_{\hat{\mathcal{R}}_{H}^{[N]}\left(z_{2}\right) H_{j}} \hat{\mathbf{P}}_{H}^{[N]}- \\
& -z_{2} \operatorname{Res}_{z=0}\left(\hat{\mathcal{R}}_{H}^{[N]}(z) \hat{\mathbf{L}}_{\hat{\mathcal{R}}_{H}^{[N]}\left(z_{2}\right) H_{j}} \hat{\boldsymbol{\mathcal { R }}}_{H}^{[N]}(z)\right)+
\end{aligned}
$$

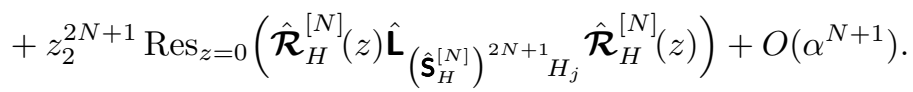

Коэффициент в этом выражении при $z_{2}^{0}$ есть

$$
\begin{aligned}
\left(\frac{\partial}{\partial \alpha} \hat{\mathbf{P}}_{H}\right)^{[N]}= & \hat{\mathbf{P}}_{H}^{[N]} \hat{\mathbf{L}}_{\hat{\mathbf{S}}_{H}^{[N]} H_{j}}-\hat{\mathbf{L}}_{\hat{\mathbf{S}}_{H}^{[N]} H_{j}} \hat{\mathbf{P}}_{H}^{[N]}+ \\
& +\operatorname{Res}_{z=0}\left(\hat{\boldsymbol{R}}_{H}^{[N]}(z) \hat{\mathbf{L}}_{\hat{\mathbf{P}}_{H} H_{j}} \hat{\boldsymbol{R}}_{H}^{[N]}(z)\right)+O\left(\alpha^{N+1}\right) .
\end{aligned}
$$

Покажем, что последний вычет тоже имеет порядок $O\left(\alpha^{N+1}\right)$. Рассмотрим коэффициент при $z_{2}^{-1}$ в (47):

$$
\begin{aligned}
0= & \hat{\boldsymbol{\mathcal { R }}}_{H}^{[N]}(z) \hat{\mathbf{L}}_{\hat{\mathbf{P}}_{H}^{[N]} H_{j}}-\hat{\mathbf{L}}_{\hat{\mathbf{P}}_{H}^{[N]} H_{j}} \hat{\boldsymbol{\mathcal { R }}}_{H}^{[N]}(z)+\hat{\boldsymbol{\mathcal { R }}}_{H}^{[N]}(z) \hat{\mathbf{L}}_{\hat{\mathbf{D}}_{H}^{[N]} H_{j}} \hat{\boldsymbol{\mathcal { R }}}_{H}^{[N]}(z)+ \\
& +z^{2 N+1}\left(\left(\hat{\boldsymbol{S}}_{H}^{[N]}\right)^{2 N+1} \hat{\mathbf{L}}_{\hat{\mathbf{P}}_{H}^{[N]} H_{j}} \hat{\boldsymbol{\mathcal { R }}}_{H}^{[N]}(z)-\hat{\boldsymbol{\mathcal { R }}}_{H}^{[N]}(z) \hat{\mathbf{L}}_{\hat{\mathbf{P}}_{H}^{[N]} H_{j}}\left(\hat{\mathbf{S}}_{H}^{[N]}\right)^{2 N+1}\right)+O\left(\alpha^{N+1}\right) .
\end{aligned}
$$


Следовательно,

$$
\begin{aligned}
\operatorname{Res}_{z=0}\left(\hat{\mathcal{R}}_{H}^{[N]}(z) \hat{\mathbf{L}}_{\hat{\mathbf{P}}_{H}^{[N]} H_{j}} \hat{\mathcal{R}}_{H}^{[N]}(z)\right)=\operatorname{Res}_{z=0}\left(\hat{\mathbf{L}}_{\hat{\mathbf{P}}_{H}^{[N]} H_{j}}\left(\hat{\mathcal{R}}_{H}^{[N]}(z)\right)^{2}\right)- \\
\quad-\operatorname{Res}_{z=0}\left(\hat{\mathcal{R}}_{H}^{[N]}(z) \hat{\mathbf{L}}_{\hat{\mathbf{D}}_{H}^{[N]} H_{j}}\left(\hat{\mathcal{R}}_{H}^{[N]}(z)\right)^{2}\right)- \\
\quad-\operatorname{Res}_{z=0}\left(z ^ { 2 N + 1 } \left(\left(\hat{\mathbf{S}}_{H}^{[N]}\right)^{2 N+1} \hat{\mathbf{L}}_{\hat{\mathbf{P}}_{H}^{[N]} H_{j}} \hat{\boldsymbol{\mathcal { R }}}_{H}^{[N]}(z)-\right.\right. \\
\left.\left.\quad-\hat{\mathcal{R}}_{H}^{[N]}(z) \hat{\mathbf{L}}_{\hat{\mathbf{P}}_{H}^{[N]} H_{j}}\left(\hat{\mathbf{S}}_{H}^{[N]}\right)^{2 N+1}\right) \hat{\boldsymbol{\mathcal { R }}}_{H}^{[N]}(z)\right)+O\left(\alpha^{N+1}\right)= \\
=-\operatorname{Res}_{z=0}\left(\hat{\mathcal{R}}_{H}^{[N]}(z) \hat{\mathbf{L}}_{\hat{\mathbf{D}}_{H}^{[N]} H_{j}}\left(\hat{\mathcal{R}}_{H}^{[N]}(z)\right)^{2}\right)+O\left(\alpha^{N+1}\right) .
\end{aligned}
$$

Мы можем продолжать этот процесс, используя коэффициенты при $z_{2}^{-2}, z_{2}^{-3}, \ldots$ В (47):

$$
\begin{aligned}
& \operatorname{Res}_{z=0}\left(\hat{\mathcal{R}}_{H}^{[N]}(z) \hat{\mathbf{L}}_{\hat{\mathbf{D}}_{H}^{[N]} H_{j}}\left(\hat{\mathcal{R}}_{H}^{[N]}(z)\right)^{2}\right)=-\operatorname{Res}_{z=0}\left(\hat{\mathcal{R}}_{H}^{[N]}(z) \hat{\mathbf{L}}_{\left(\hat{\mathbf{D}}_{H}^{[N]}\right)^{2} H_{j}}\left(\hat{\mathcal{R}}_{H}^{[N]}(z)\right)^{3}\right)+ \\
& +O\left(\alpha^{N+1}\right)=\cdots=(-1)^{N+1} \operatorname{Res}_{z=0}\left(\hat{\mathcal{R}}_{H}^{[N]}(z) \hat{\mathbf{L}}_{\left(\hat{\mathbf{D}}_{H}^{[N]}\right)^{N} H_{j}}\left(\hat{\boldsymbol{\mathcal { R }}}_{H}^{[N]}(z)\right)^{N+1}\right)+ \\
& +O\left(\alpha^{N+1}\right)=O\left(\alpha^{N+1}\right) .
\end{aligned}
$$

Подстановка этой цепочки в (48) завершает доказательство того, что проектор преобразуется канонически:

$$
\left(\frac{\partial}{\partial \alpha} \hat{\mathbf{P}}_{H}\right)^{[N]}=\hat{\mathbf{L}}_{-\hat{\mathbf{S}}_{H}^{[N]} H_{j}} \hat{\mathbf{P}}_{H}^{[N]}-\hat{\mathbf{P}}_{H}^{[N]} \hat{\mathbf{L}}_{-\hat{\mathbf{S}}_{H}^{[N]} H_{j}}+O\left(\alpha^{N+1}\right)
$$

с генератором $-\hat{\mathbf{S}}_{H}^{[N]} H_{j}$ в любом порядке теории возмущений.

\section{“Квадратный” алгоритм}

Выражение (41) приводит к эффективному вычислительному алгоритму для генератора $G^{[N]}=-\hat{\mathbf{S}}_{H}^{[N]} H_{j}$. Рассмотрим квадратную таблицу

$$
\begin{array}{lcccc}
F_{0}^{0}(\mathbf{x}) & F_{0}^{1}(\mathbf{x}) & F_{0}^{2}(\mathbf{x}) & \ldots & F_{0}^{N+1}(\mathbf{x}) \\
F_{1}^{0}(\mathbf{x}) & F_{1}^{1}(\mathbf{x}) & F_{1}^{2}(\mathbf{x}) & \ldots & F_{1}^{N+1}(\mathbf{x}) \\
\ldots \ldots \ldots \ldots \ldots \ldots \ldots \ldots \ldots \ldots \ldots \ldots \ldots \ldots \ldots \ldots & \ldots \ldots \ldots \\
F_{N-1}^{0}(\mathbf{x}) & F_{N-1}^{1}(\mathbf{x}) & F_{N-1}^{2}(\mathbf{x}) & \ldots & F_{N-1}^{N+1}(\mathbf{x}) \\
& & & & \\
& & & & \\
& & &
\end{array}
$$

Здесь первая строка состоит из следующих элементов:

$$
F_{0}^{0}(\mathbf{x})=\hat{\mathbf{Z}}_{0}^{0} H_{j}=\hat{\mathbf{P}} H_{j}, \quad F_{0}^{m}(\mathbf{x})=\hat{\mathbf{Z}}_{0}^{m} H_{j}=-\hat{\mathbf{S}}^{m} H_{j}
$$


а каждая следующая строка получается из предыдущей по правилу

$$
F_{n+1}^{m}(\mathbf{x})=\sum_{k=0}^{m} \hat{\mathbf{Z}}_{0}^{m-k} \hat{\mathbf{L}}_{H_{j}} F_{n}^{k}(\mathbf{x}) .
$$

Усеченный нормализующий генератор дается выражением $G^{[N]}=\sum_{n=0}^{N} \alpha^{n} F_{n}^{n+1}(\mathbf{x})$. Очевидной оптимизацией вычислений является использование величин $\hat{\mathbf{L}}_{H_{j}} F_{n}^{k}(\mathbf{x})$.

Благодарности. Автор искренне благодарен профессору С. В. Клименко, оживившему мой интерес к этой работе, директору Центра технической поддержки РДТЕХ С.П. Мисюре за многолетнюю поддержку исследований и профессору А. С. Холево.

\section{Список литературы}

[1] J. R. Cary, Phys. Rep., 79:2 (1981), 129-159.

[2] А. Мессиа, Квантовая механика, 1, Наука, М., 1978.

[3] А. Пуанкаре, Избранные труды в 3-х т., т. 1: Новые методы небесной механики, Наука, M., 1971.

[4] A. Deprit, Celestial Mech., 1:1 (1969), 12-30.

[5] A. J. Dragt, J. M. Finn, J. Math. Phys., 17:12 (1976), 2215-2227.

[6] J. H. Van Vleck, Phys. Rev., 33:4 (1929), 467-506.

[7] I. Shavitt, L. T. Redmon, J. Chem. Phys., 73:11 (1980), 5711-5717.

[8] D. J. Klein, J. Chem. Phys., 61:3 (1974), 786-798.

[9] Д. Д. Биркгоф, Динамические системы, РХД, Ижевск, 1999.

[10] F. G. Gustavson, Astron. J., 71 (1966), 670.

[11] M. Degli Esposti, S. Graffi, J. Herczynski, Ann. Phys., 209:2 (1991), 364-392.

[12] A. S. Nikolaev, J. Math. Phys., 37:6 (1996), 2643-2661.

[13] Т. Като, Теория возмущений линейнъх операторов, Мир, М., 1972.

[14] H. Spohn, Phys. A: Statist. Mech. Appl., 80:4 (1975), 323-338.

[15] А. Н. Колмогоров, Докл. АН СССР, 93 (1953), 763-766.

[16] В. И. Арнольд, УМН, 18:6(114) (1963), 91-192.

[17] S. Ferraz-Mello, Canonical Perturbation Theories: Degenerate Systems and Resonance, Springer, Berlin, 2007.

[18] Р. Балеску, Равновесная и неравновесная статистическая механика, Мир, М., 1978.

[19] А.С. Николаев, Kato perturbation expansion in classical mechanics and an explicit expression for a Deprit generator, 2015, http://andreysnikolaev.wordpress.com/demo.

[20] J. Kuipers, T. Ueda, J. A. M. Vermaseren, J. Vollinga, Comput. Phys. Commun., 184:5 (2013), 1453-1467, arXiv: 1203.6543.

[21] В. И. Арнольд, Математические методы классической механики, Наука, М., 1989.

[22] A. Giorgilli, On the representation of maps by lie transforms, arXiv: 1211.5674.

[23] P. V. Koseleff, Celestial Mech. Dynam. Astron., 58:1 (1994), 17-36.

[24] G. Hori, J. Japan Astron. Soc., 18 (1966), 287.

[25] Э. Хилле, Р. Филлипс, Функииональный анализ и полугруппъ, ИЛ, М., 1962.

[26] Ю.А. Митропольский, Метод усреднения в нелинейной механике, Наукова думка, Киев, 1971.

[27] Н.Н.Боголюбов, Ю.А. Митропольский, Асимптотические методъ в теории нелинейных колебаний, Гостехиздат, М., 1955. 
[28] J. A. Sanders, F. Verhulst, J. Murdock, Averaging Methods in Nonlinear Dynamical Systems, Springer, New York, 2007.

[29] К.О. Фридрихс, Возмущение спектра операторов в гильбертовом пространстве, Мир, М., 1969.

[30] R. Cushman, "Normal form for Hamiltonian vectorfields with periodic flow", Differential Geometric Methods in Mathematical Physics (Jerusalem, August 5-11, 1982), Mathematical Physics Studies, 6, ed. S. Sternberg, Reidel Publ., Dordrecht, Boston, Lancaster, 1984, $125-144$.

[31] M. Avendaño Camacho, Y. M. Vorobiev, Russ. J. Math. Phys., 18 (2011), 243-257.

[32] А.Д. Брюно, Локальный метод нелинейного анализа дифференциальных уравнений, Наука, М., 1979.

[33] Ю.А. Митропольский, А. К. Лопатин, Теоретико-групповой подход в асимптотических методах нелинейной механики, Наукова думка, Киев, 1988.

[34] В. Н. Богаевский, А. Я. Повзнер, Алгебраические методы в нелинейной теории возмущений, Наука, М., 1987.

[35] В. Ф. Журавлёв, ПММ, 66:3 (2002), 356-365.

[36] J. Awrejcewicz, A. G. Petrov, Nonlinear Dynam., 48:1-2 (2007), 185-197.

[37] А. Х. Найфэ, Методъ возмущений, Мир, М., 1976.

[38] Г. Е. О. Джакалья, Методы теории возмущений для нелинейных систем, Наука, М., 1979.

[39] Ю. К. Мозер, УМH, 24:2(146) (1969), 165-211.

[40] A.S. Nikolaev, J. Phys. A: Math. Gen., 28:15 (1995), 4407-4414.

[41] Э. Л. Бурштейн, Л. С. Соловьев, Докл. АН СССР, 139:4 (1961), 855-858.

[42] H. Primas, Rev. Modern Phys., 35:3 (1963), 710-711.

[43] Vl. G. Tyuterev, V. I. Perevalvo, Chem. Phys. Lett., 74:3 (1980), 494-502.

[44] H. R. Jauslin, S. Guérin, S. Thomas, Phys. A: Statist. Mech. Appl., 279:1-4 (2000), 432-442.

[45] А.С. Николаев, "Разложение Като в теории возмущений классической механики", Труды Международной конферениии по физико-технической информатике СРТ-2013 (12-19 мая 2013 г., Ларнака, Кипр), ред. С. В. Клименко, ИФТИ, Протвино, 2013, 1-12.

[46] M. Vittot, "A simple and compact presentation of Birkhoff series", Nonlinear Evolution and Chaotic Phenomena (Noto, June 8-19, 1987), NATO Advanced Science Institutes Series B, 176, eds. G. Gallavotti, P. F. Zweifel, Plenum Press, New York, 1988, 173-183.

[47] G. Contopoulos, C. Efthymiopoulos, A. Giorgilli, J. Phys. A: Math. Gen., 36:22 (2003), 8639-8660.

[48] C. Jaffe, W. P. Reinhardt, J. Chem. Phys., 77:10 (1982), 5191-5203.

[49] D. Treschev, O. Zubelevich, Introduction to the Perturbation Theory of Hamiltonian Systems, Berlin, 2010.

[50] L. H. Eliasson, Math. Phys. Electronic J., 2 (1996), 4, 33 pp.

[51] G. Gallavotti, Rev. Math. Phys., 6:3 (1994), 343-411.

[52] A. Kiper, Math. Comp., 43:67 (1984), 247-259.

Поступила в редакцию 9.07.2014, после доработки 28.08.2014 